Document downloaded from:

http://hdl.handle.net/10251/107452

This paper must be cited as:

Albanese, A.; Bonet Solves, JA.; Ricker, WJ. (2018). The Cesàro operator on Korenblum type spaces of analytic functions. Collectanea mathematica. 69(2):263-281. doi:10.1007/s13348-017-0205-7

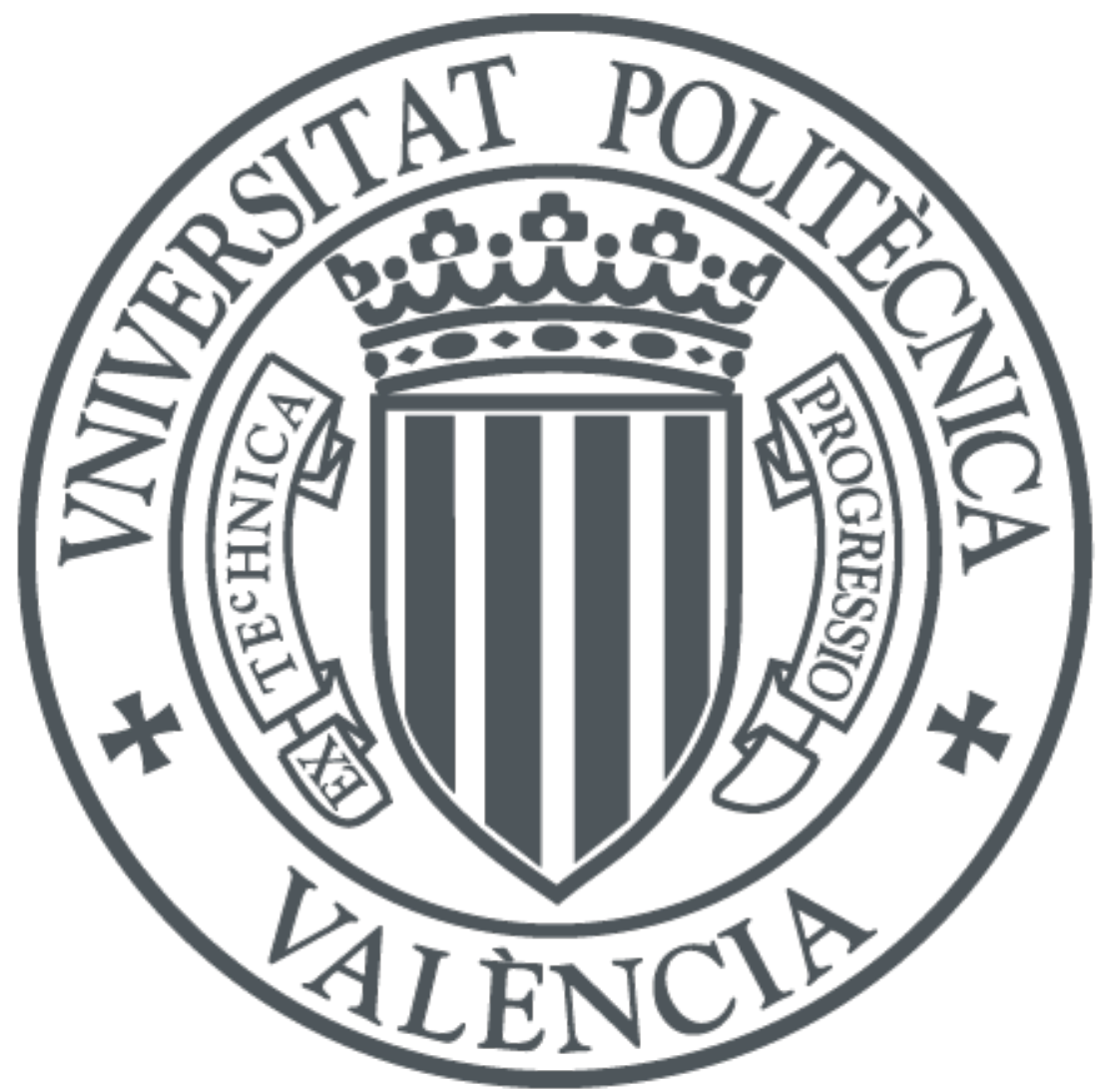

The final publication is available at

http://doi.org/10.1007/s13348-017-0205-7

Copyright Springer-Verlag

Additional Information 


\title{
THE CESÀRO OPERATOR ON KORENBLUM TYPE SPACES OF ANALYTIC FUNCTIONS
}

\author{
ANGELA A. ALBANESE, JOSÉ BONET, WERNER J. RICKER
}

\begin{abstract}
The spectrum of the Cesàro operator $\mathrm{C}$, which is always continuous (but never compact) when acting on the classical Korenblum space and other related weighted Fréchet or (LB) spaces of analytic functions on the open unit disc, is completely determined. It turns out that such spaces are always Schwartz but, with the exception of the Korenblum space, never nuclear. Some consequences concerning the mean ergodicity of $\mathrm{C}$ are deduced.
\end{abstract}

\section{INTRODUCTION AND MAIN RESULTS.}

Let $H(\mathbb{D})$ denote the Fréchet space of all analytic functions $f: \mathbb{D} \rightarrow \mathbb{C}$ equipped with the topology of uniform convergence on the compact subsets of $\mathbb{D}:=\{z \in \mathbb{C}:|z|<1\}$. The classical Cesàro operator $C$ is given by

$$
f \mapsto \mathrm{C}(f): z \mapsto \frac{1}{z} \int_{0}^{z} \frac{f(\zeta)}{1-\zeta} d \zeta, \quad z \in \mathbb{D} \backslash\{0\}, \quad \text { and } \quad \mathrm{C}(f)(0)=f(0),
$$

for $f \in H(\mathbb{D})$. It is a Fréchet space isomorphism of $H(\mathbb{D})$ onto itself. In terms of the Taylor coefficients $\widehat{f}(n):=\frac{f^{(n)}(0)}{n !}$, for $n \in \mathbb{N}_{0}$, of functions $f(z)=\sum_{n=0}^{\infty} \widehat{f}(n) z^{n} \in H(\mathbb{D})$ one has the description

$$
\mathrm{C}(f)(z)=\sum_{n=0}^{\infty}\left(\frac{1}{n+1} \sum_{k=0}^{n} \widehat{f}(k)\right) z^{n}, \quad z \in \mathbb{D} .
$$

The linear operator $C$ is said to act in a vector subspace $X \subseteq H(\mathbb{D})$ if it maps $X$ into itself. Of particular interest is the situation when $X$ is a Fréchet space, i.e., a complete metrizable locally convex space (in particular, a Banach space) or a countable inductive limit of Banach spaces, i.e. an (LB)-space. Two fundamental questions arise: Is $\mathrm{C}: X \rightarrow X$ continuous and, if so, what is its spectrum?

The continuity, compactness and spectrum of generalized Cesàro operators on Banach spaces of analytic functions on $\mathbb{D}$ have attracted much attention; see $[7,8,10,11]$ and the survey papers [6, 34]. Aleman and Persson $[9,31]$ investigated in detail the spectrum of generalized Cesàro

Key words and phrases. Cesàro operator, weighted spaces of analytic functions, spectrum, Fréchet spaces, (LB)-spaces, mean ergodicity.

Mathematics Subject Classification 2010: Primary 47A10, 47B38; Secondary 46A11, 46E10, 47A35, 47B10. 
operators on various spaces of analytic functions, including Hardy spaces, weighted Bergman spaces and Dirichlet spaces. For a detailed account of the development of research in this direction, we refer to the introduction of [9].

For each $\gamma>0$, the growth classes of analytic functions

$$
A^{-\gamma}:=\left\{f \in H(\mathbb{D}):\|f\|_{-\gamma}:=\sup _{z \in \mathbb{D}}(1-|z|)^{\gamma}|f(z)|<\infty\right\}
$$

and

$$
A_{0}^{-\gamma}:=\left\{f \in H(\mathbb{D}): \lim _{|z| \rightarrow 1^{-}}(1-|z|)^{\gamma}|f(z)|=0\right\}
$$

play an important role in $[9,31]$. Both are Banach spaces when endowed with the norm $\|\cdot\|_{-\gamma}$. The space $A_{0}^{-\gamma}$, which is a closed subspace of $A^{-\gamma}$, coincides with the closure of the polynomials on $A^{-\gamma},[16]$. These Banach spaces, as well as their intersections and unions, play a relevant and important role in connection with the interpolation and sampling of analytic functions; see [24, Section 4.3]. They are particular examples of weighted Banach spaces $H_{v}^{\infty}$ and $H_{v}^{0}$ of analytic functions on $\mathbb{D}$ which have been investigated by many authors since the work of Shields and Williams [33]; see, for example, $[13,14,15,16,28]$ and the references therein.

For each pair $0<\mu_{1}<\mu_{2}$ we have $A^{-\mu_{1}} \subseteq A_{0}^{-\mu_{2}}$, with the natural inclusion being continuous. Moreover, for each $\gamma>0, A^{-\gamma}$ is canonically isomorphic to the bidual Banach space $\left(A_{0}^{-\gamma}\right)^{\prime \prime}$ of $A_{0}^{-\gamma},[16,33]$. In terms of this biduality the operator $\mathrm{C}: A^{-\gamma} \rightarrow A^{-\gamma}$ is precisely the bidual operator of C: $A_{0}^{-\gamma} \rightarrow A_{0}^{-\gamma}$.

We recall the following result [9, Theorem 5.1 and Corollary 5.1], [31, Theorem 4.1] of Aleman and Persson concerning the eigenvalues and spectrum of $\mathrm{C}$ on $A_{0}^{-\gamma}$ and $A^{-\gamma}$; it will be quoted on several occasions. Further properties of $\mathrm{C}$ which complement this result occur in [3].

Theorem 1.1. Let $\gamma>0$. Both of the Cesàro operators $\mathrm{C}: A_{0}^{-\gamma} \rightarrow A_{0}^{-\gamma}$ and $\mathrm{C}: A^{-\gamma} \rightarrow A^{-\gamma}$ are continuous and satisfy the following properties.

(i) $\sigma_{p t}\left(\mathrm{C} ; A_{0}^{-\gamma}\right)=\left\{\frac{1}{m}: m \in \mathbb{N}, m<\gamma\right\}$.

(ii) $\sigma\left(\mathrm{C} ; A_{0}^{-\gamma}\right)=\sigma_{p t}\left(\mathrm{C} ; A_{0}^{-\gamma}\right) \cup\left\{\lambda \in \mathbb{C}:\left|\lambda-\frac{1}{2 \gamma}\right| \leq \frac{1}{2 \gamma}\right\}$.

(iii) Let $\left|\lambda-\frac{1}{2 \gamma}\right|<\frac{1}{2 \gamma}$ ( equivalently, $\operatorname{Re}\left(\frac{1}{\lambda}\right)>\gamma$ ). The range $\operatorname{Im}(\lambda I-\mathrm{C})$ of $\lambda I-\mathrm{C}$ is closed in $A_{0}^{-\gamma}$ and has codimension 1 in $A_{0}^{-\gamma}$.

Moreover, the spectra of $\mathrm{C}$ acting in $A^{-\gamma}$ are given as follows.

(iv) $\sigma_{p t}\left(\mathrm{C} ; A^{-\gamma}\right)=\left\{\frac{1}{m}: m \in \mathbb{N}, m \leq \gamma\right\}$.

(v) $\sigma\left(\mathrm{C} ; A^{-\gamma}\right)=\sigma\left(\mathrm{C} ; A_{0}^{-\gamma}\right)$.

The purpose of this paper is to investigate the Cesàro operator C in spaces that arise as unions or as intersections of growth spaces defined as follows for each $\gamma \geq 0$. We begin with the (decreasing) intersection 


$$
A_{+}^{-\gamma}:=\cap_{\mu>\gamma} A^{-\mu}=\left\{f \in H(\mathbb{D}): \sup _{z \in \mathbb{D}}(1-|z|)^{\mu}|f(z)|<\infty \forall \mu>\gamma\right\},
$$

in which case also

$$
A_{+}^{-\gamma}=\cap_{\mu>\gamma} A_{0}^{-\mu}=\left\{f \in H(\mathbb{D}): \lim _{|z| \rightarrow 1^{-}}(1-|z|)^{\mu}|f(z)|=0 \forall \mu>\gamma\right\} .
$$

Then $A_{+}^{-\gamma}$ is a Fréchet space when endowed with the lc-topology generated by the increasing sequence of norms $\|f\|_{k}:=\sup _{z \in \mathbb{D}}(1-|z|)^{\gamma+\frac{1}{k}}|f(z)|$, for $f \in A_{+}^{-\gamma}$ and each $k \in \mathbb{N}$. We note, for $0<\mu_{1}<\mu_{2}$, that the natural inclusion $A^{-\mu_{1}} \subseteq A_{0}^{-\mu_{2}}$ is actually a compact operator between Banach spaces. This follows from [17, Theorem 3.3], for example, if we substitute there $\varphi(z):=z$, for $z \in \mathbb{D}$, with $v(z):=(1-|z|)^{\mu_{1}}$ and $w(z):=(1-|z|)^{\mu_{2}}$ (in which case $v=\widetilde{v}$ ) satisfying $\lim _{|z| \rightarrow 1^{-}} v(z)=\lim _{|z| \rightarrow 1^{-}} w(z)=0$ and observe that $H_{v}^{\infty}=A^{-\mu_{1}}$ (resp. $\left.H_{w}^{\infty}=A^{-\mu_{2}}\right)$ and $H_{v}^{0}=A_{0}^{-\mu_{1}}\left(\right.$ resp. $H_{w}^{0}=A_{0}^{-\mu_{2}}$ ). Consequently, $A_{+}^{-\gamma}$ is a Fréchet Schwartz space, [25, §21.1 Example 1(b)]. In particular, bounded subsets of $A_{+}^{-\gamma}$ are relatively compact as $A_{+}^{-\gamma}$ is also a Fréchet Montel space, [29, Remark 24.24]. Moreover, for every $\mu>\gamma>0$ we have $A^{-\gamma} \subseteq A_{+}^{-\gamma} \subseteq A_{0}^{-\mu}$ with continuous inclusions. Since $A_{+}^{-\gamma}$ contains the polynomials, it is dense in $A_{0}^{-\mu}$ for every $\mu>\gamma$. In addition, since evaluations at points of $\mathbb{D}$ are continuous linear functionals on each Banach space $A_{0}^{-\mu}$ for $\mu>0$, [33, Lemma 1], they are also continuous on $A_{+}^{-\gamma}$ for each $\gamma \geq 0$.

Consider now the (increasing) union

$$
A_{-}^{-\gamma}:=\cup_{\mu<\gamma} A^{-\mu}=\left\{f \in H(\mathbb{D}): \sup _{z \in \mathbb{D}}(1-|z|)^{\mu}|f(z)|<\infty \text { for some } \mu<\gamma\right\}
$$

in which case also

$$
A_{-}^{-\gamma}=\cup_{\mu<\gamma} A_{0}^{-\mu}=\left\{f \in H(\mathbb{D}): \lim _{|z| \rightarrow 1^{-}}(1-|z|)^{\mu}|f(z)|=0 \text { for some } \mu<\gamma\right\} \text {, }
$$

for each $0<\gamma \leq \infty$. Each space $A_{-}^{-\gamma}$ is endowed with the finest lctopology such that all the natural inclusion maps $A^{-\mu} \subseteq A_{-}^{-\gamma}$, for $\mu<\gamma$, are continuous. In particular, since $A^{-\mu_{1}} \subseteq A^{-\mu_{2}}$ compactly whenever $0<\mu_{1}<\mu_{2}$, the space $A_{-}^{-\gamma}=\cup_{k \in \mathbb{N}} A^{-\left(\gamma-\frac{1}{k}\right)}$ is the complete (DFS)-space

$$
A_{-}^{-\gamma}:=\operatorname{ind}_{k} A^{-\left(\gamma-\frac{1}{k}\right)}=\operatorname{ind}_{k} A_{0}^{-\left(\gamma-\frac{1}{k}\right)},
$$

which is necessarily a Schwartz space, [29, Proposition 25.20]. Of course, the inductive limit is taken over all $k \in \mathbb{N}$ such that $\left(\gamma-\frac{1}{k}\right)>0$. Fürthermore, the (LB)-space $A_{-}^{-\gamma}$ is regular, i.e., every bounded set $B \subseteq A_{-}^{-\gamma}$ is contained and bounded in the Banach space $A^{-\mu}$ for some $0<\mu<\gamma$.

The well known Korenblum space $A_{-}^{-\infty}$, [26], denoted simply by $A^{-\infty}$, is defined via

$$
A^{-\infty}:=\cup_{0<\gamma<\infty} A^{-\gamma}=\cup_{n \in \mathbb{N}} A^{-n}
$$


and is endowed with the finest lc-topology such that all the natural inclusion maps $A^{-n} \subseteq A^{-\infty}$ are continuous, that is, $A^{-\infty}=\operatorname{ind}_{n} A^{-n}$. Again the inductive limit is regular and $A^{-\infty}$ is a Schwartz space. Further information can be found in [24, Section 4.3]. We mention, for all $\gamma>0$, that $A_{-}^{-\gamma} \subseteq$ $A_{0}^{-\gamma} \subseteq A^{-\gamma} \subseteq A_{+}^{-\gamma}$ with continuous inclusions. In particular, all evaluations at points of $\mathbb{D}$ are continuous linear functionals on each space $A_{-}^{-\gamma}$, for $0<$ $\gamma \leq \infty$. Since the polynomials lie in $A_{-}^{-\gamma}$ for all $0<\gamma \leq \infty$, it is clear that $A_{-}^{-\gamma}$ is dense in $A_{0}^{-\gamma}$ for all $0<\gamma<\infty$. Each Cesàro operator $\mathrm{C}: A_{-}^{-\gamma} \rightarrow$ $A_{-}^{-\gamma}$ (and $\mathrm{C}: A_{+}^{-\gamma} \rightarrow A_{+}^{-\gamma}$ ) is continuous because, by Theorem 1.1, it acts continuously in each step. Since every closed linear map between (LF)spaces is continuous, it follows that all the natural inclusions $A_{+}^{-\gamma} \subseteq H(\mathbb{D})$ for $\gamma \geq 0$ and $A_{-}^{-\gamma} \subseteq H(\mathbb{D})$ for $0<\gamma \leq \infty$ are continuous.

Let $X$ be a locally convex Hausdorff space (briefly, lcHs) and $\Gamma_{X}$ a system of continuous seminorms determining the topology of $X$. Let $X^{\prime}$ denote the space of all continuous linear functionals on $X$. The family of all bounded subsets of $X$ is denoted by $\mathcal{B}(X)$. Denote the identity operator on $X$ by $I$. Let $\mathcal{L}(X)$ denote the space of all continuous linear operators from $X$ into itself. For $T \in \mathcal{L}(X)$, the resolvent set $\rho(T)$ of $T$ consists of all $\lambda \in \mathbb{C}$ such that $R(\lambda, T):=(\lambda I-T)^{-1}$ exists in $\mathcal{L}(X)$. The set $\sigma(T):=\mathbb{C} \backslash \rho(T)$ is called the spectrum of $T$. The point spectrum $\sigma_{p t}(T)$ of $T$ consists of all $\lambda \in \mathbb{C}$ such that $(\lambda I-T)$ is not injective. If we need to stress the space $X$, then we also write $\sigma(T ; X), \sigma_{p t}(T ; X)$ and $\rho(T ; X)$. Given $\lambda, \mu \in \rho(T)$ the resolvent identity $R(\lambda, T)-R(\mu, T)=(\mu-\lambda) R(\lambda, T) R(\mu, T)$ holds. Unlike for Banach spaces, it may happen that $\rho(T)=\emptyset$ or that $\rho(T)$ is not open in $\mathbb{C}$. This is why some authors prefer the subset $\rho^{*}(T)$ of $\rho(T)$ consisting of all $\lambda \in \mathbb{C}$ for which there exists $\delta>0$ such that $B(\lambda, \delta):=\{z \in \mathbb{C}:|z-\lambda|<$ $\delta\} \subseteq \rho(T)$ and $\{R(\mu, T): \mu \in B(\lambda, \delta)\}$ is equicontinuous in $\mathcal{L}(X)$. If $X$ is a Fréchet space or even an (LF)-space, then it suffices that such sets are bounded in $\mathcal{L}_{s}(X)$, where $\mathcal{L}_{s}(X)$ denotes $\mathcal{L}(X)$ endowed with the strong operator topology $\tau_{s}$ which is determined by the seminorms $T \mapsto q_{x}(T):=$ $q(T x)$, for all $x \in X$ and $q \in \Gamma_{X}$. The advantage of $\rho^{*}(T)$, whenever it is non-empty, is that it is open and the resolvent map $R: \lambda \mapsto R(\lambda, T)$ is analytic from $\rho^{*}(T)$ into $\mathcal{L}_{b}(X),\left[2\right.$, Proposition 3.4]. Here $\mathcal{L}_{b}(X)$ denotes $\mathcal{L}(X)$ endowed with the topology $\tau_{b}$ of uniform convergence on members of $\mathcal{B}(X)$; it is determined by the seminorms $T \mapsto q_{B}(T):=\sup _{x \in B} q(T x)$, for all $B \in \mathcal{B}(X)$ and $q \in \Gamma_{X}$. Define $\sigma^{*}(T):=\mathbb{C} \backslash \rho^{*}(T)$, which is a closed set containing $\sigma(T)$. If $T \in \mathcal{L}(X)$ with $X$ a Banach space, then $\sigma(T)=\sigma^{*}(T)$. In [2, Remark 3.5(vi), p.265] an example of a continuous linear operator $T$ on a Fréchet space $X$ is presented such that $\overline{\sigma(T)} \subset \sigma^{*}(T)$ properly. For undefined concepts concerning lcHs', see [25, 29]. We introduce the notation $\Sigma:=\left\{\frac{1}{m}: m \in \mathbb{N}\right\}$ and $\Sigma_{0}:=\{0\} \cup \Sigma$.

The first main result of this paper is the following one.

Theorem 1.2. (a) Let $\gamma \in(0, \infty)$. 
(i) $\sigma_{p t}\left(\mathrm{C} ; A_{+}^{-\gamma}\right)=\left\{\frac{1}{m}: m \in \mathbb{N}, m \leq \gamma\right\}$.

(ii) $\sigma\left(\mathrm{C} ; A_{+}^{-\gamma}\right)=\{0\} \cup \sigma_{p t}\left(\mathrm{C} ; A_{+}^{-\gamma}\right) \cup\left\{\lambda \in \mathbb{C}:\left|\lambda-\frac{1}{2 \gamma}\right|<\frac{1}{2 \gamma}\right\}$.

(iii) $\sigma^{*}\left(\mathrm{C} ; A_{+}^{-\gamma}\right)=\overline{\sigma\left(\mathrm{C} ; A_{+}^{-\gamma}\right)}$.

(b) $\operatorname{Let} \gamma=0$.

(i) $\sigma_{p t}\left(\mathrm{C} ; A_{+}^{-0}\right)=\emptyset$.

(ii) $\sigma\left(\mathrm{C} ; A_{+}^{-0}\right)=\{0\} \cup\{\lambda \in \mathbb{C}: \operatorname{Re}(\lambda)>0\}$.

(iii) $\sigma^{*}\left(\mathrm{C} ; A_{+}^{-0}\right)=\overline{\sigma\left(\mathrm{C} ; A_{+}^{-0}\right)}$.

Observe that the spectra $\sigma\left(\mathrm{C} ; A_{+}^{-\gamma}\right)$, for $\gamma \in(0, \infty)$, are bounded but not closed sets in $\mathbb{C}$ and that $\sigma_{p t}\left(\mathrm{C} ; A_{+}^{-\gamma}\right)=\emptyset$ with $\sigma\left(\mathrm{C} ; A_{+}^{-\gamma}\right)=\{0\} \cup\{\lambda \in \mathbb{C}$ : $\left.\left|\lambda-\frac{1}{2 \gamma}\right|<\frac{1}{2 \gamma}\right\}$ whenever $0<\gamma<1$. Moreover, $\sigma\left(\mathrm{C} ; A_{+}^{-0}\right)$ is unbounded and not closed in $\mathbb{C}$.

The second main result is the following one.

Theorem 1.3. (a) Let $\gamma \in(0, \infty)$.

(i) $\sigma_{p t}\left(\mathrm{C} ; A_{-}^{-\gamma}\right)=\left\{\frac{1}{m}: m \in \mathbb{N}, m<\gamma\right\}$.

(ii) $\sigma\left(\mathrm{C} ; A_{-}^{-\gamma}\right)=\sigma_{p t}\left(\mathrm{C} ; A_{-}^{-\gamma}\right) \cup\left\{\lambda \in \mathbb{C}:\left|\lambda-\frac{1}{2 \gamma}\right| \leq \frac{1}{2 \gamma}\right\}$.

(iii) $\sigma^{*}\left(\mathrm{C} ; A_{-}^{-\gamma}\right)=\sigma\left(\mathrm{C} ; A_{-}^{-\gamma}\right)$.

(b) For the Korenblum space $A^{-\infty}$ the following properties hold.

(i) $\sigma\left(\mathrm{C} ; A^{-\infty}\right)=\sigma_{p t}\left(\mathrm{C} ; A^{-\infty}\right)=\Sigma$.

(ii) $\sigma^{*}\left(\mathrm{C} ; A^{-\infty}\right)=\Sigma_{0}$.

Note that the spectra $\sigma\left(\mathrm{C} ; A_{-}^{-\gamma}\right)$, for $0<\gamma<\infty$, are compact sets in $\mathbb{C}$ and that $\sigma_{p t}\left(\mathrm{C} ; A_{-}^{-\gamma}\right)=\emptyset$ with $\sigma\left(\mathrm{C} ; A_{-}^{-\gamma}\right)=\left\{\lambda \in \mathbb{C}:\left|\lambda-\frac{1}{2 \gamma}\right| \leq \frac{1}{2 \gamma}\right\}$ whenever $0<\gamma \leq 1$. In the Korenblum space $A^{-\infty}$ the spectrum of $\mathrm{C}$ is precisely $\Sigma$ and hence, it is bounded but not compact in $\mathbb{C}$.

Recall that an operator $T \in \mathcal{L}(X)$, with $X$ a lcHs, is compact (resp. weakly compact) if there exists a neighbourhood $U$ of 0 such that $T(U)$ is a relatively compact (resp. relatively weakly compact) subset of $X$. If $T$ is compact, then $\sigma(T ; X)$ is a compact set in $\mathbb{C}$ and every non-zero point of $\sigma(T ; X)$ is isolated, [22, Theorem 9.10.2], [23, p.204]. This fact, together with Theorems 1.2 and 1.3 , yields the following result.

Proposition 1.4. The Cesàro operator $\mathrm{C}$ fails to be compact in the Frèchet spaces $A_{+}^{-\gamma}$ for $0 \leq \gamma<\infty$, in the (DFS)-spaces $A_{-}^{-\gamma}$ for $0<\gamma<\infty$, and in the Korenblum space $A^{-\infty}$.

Since the spaces $A_{+}^{-\gamma}$ for $0 \leq \gamma<\infty$ and $A_{-}^{-\gamma}$ for $0<\gamma \leq \infty$ are all Montel, there is no distinction between weakly compact and compact operators in these spaces, i.e., $\mathrm{C}$ also fails to be weakly compact in all of these spaces.

Section 2 is devoted to the proofs of Theorem 1.2 and Theorem 1.3. An application to the operator of differentiation is given in Proposition 2.10. 
Section 3 determines the mean ergodic properties of $\mathrm{C}$ when acting in the spaces $A_{+}^{-\gamma}$ (resp. $A_{-}^{-\gamma}$ ); these properties depend critically on whether $\gamma<1$ or $\gamma \geq 1$ (resp. $\gamma \leq 1$ or $\gamma>1$ ); see Propositions 3.3 and 3.4.

Both the Korenblum space $A^{-\infty}$ and its ambient space $H(\mathbb{D})$ are known to be nuclear. In Section 4 it is shown that this situation is special: the spaces $A_{+}^{-\gamma}$ for $0 \leq \gamma<\infty$ and $A_{-}^{-\gamma}$ for $0<\gamma<\infty$ all fail to be nuclear.

In an Appendix at the end of the paper we present two abstract results (one from [4] and the other one new) concerning the spectra of operators in Fréchet spaces and in inductive limits of Banach spaces, which are used on various occasions in Section 2.

\section{Proofs of the MAIN RESUlts}

We begin immediately with the proof of Theorem 1.2, which is obtained as a consequence of several propositions.

Proposition 2.1. Let $0 \leq \gamma<\infty$. Then

$$
\sigma_{p t}\left(\mathrm{C} ; A_{+}^{-\gamma}\right)=\left\{\frac{1}{m}: m \in \mathbb{N}, m \leq \gamma\right\} .
$$

In particular, $\sigma_{p t}\left(\mathrm{C} ; A_{+}^{-\gamma}\right)=\emptyset$ whenever $0 \leq \gamma<1$.

Proof. Fix $\lambda \in \sigma_{p t}\left(\mathrm{C} ; A_{+}^{-\gamma}\right)$. Then there exists $f \in A_{+}^{-\gamma}$ satisfying $\mathrm{C} f=\lambda f$. Applying Theorem 1.1(i) for $(\gamma+1)>0$ in place of $\gamma$ and noting $f \in A_{0}^{-(\gamma+1)}$ it follows that $\lambda=\frac{1}{m}$ for some unique $m \in \mathbb{N}$ with $m<(\gamma+1)$. But, for every $\mu>\gamma$ we also have that $f \in A_{0}^{-\mu}$ with $\mathrm{C} f=\frac{1}{m} f$ in $A_{0}^{-\mu}$. Again by Theorem 1.1(i), now applied to $\mu$ in place of $\gamma$, it follows that $\frac{1}{m}<\mu$ holds for every $\mu>\gamma$. Accordingly, $\lambda$ belongs to the right-side of (2.1). On the other hand, if $\lambda=\frac{1}{m}$ with $m \in \mathbb{N}$ satisfying $m \leq \gamma$, then by Theorem 1.1(iv) there exists $f \in A^{-\gamma} \subseteq A_{+}^{-\gamma}$ satisfying $\mathrm{C} f=\lambda f$. Therefore, $\lambda \in \sigma_{p t}\left(\mathrm{C} ; A_{+}^{-\gamma}\right)$.

This argument implies that $\sigma_{p t}\left(\mathrm{C} ; A_{+}^{-\gamma}\right)=\emptyset$ for $0 \leq \gamma<1$.

For $\varphi \in H^{\infty}(\mathbb{D})$ let $M_{\varphi}$ denote the operator in $H(\mathbb{D})$ of multiplication by $\varphi$, in which case $M_{\varphi} \in \mathcal{L}(H(\mathbb{D}))$. The differentiation operator $D: f \mapsto f^{\prime}$, for $f \in H(\mathbb{D})$, also belongs to $\mathcal{L}(H(\mathbb{D})$ ). It then follows from (1.1) that $\mathrm{C}^{-1} \in \mathcal{L}(H(\mathbb{D}))$ is given by $\mathrm{C}^{-1}=M_{1-z} D M_{z}$, i.e., for all $g \in H(\mathbb{D})$ we have

$$
\mathrm{C}^{-1} g(z)=(1-z)\left(g(z)+z g^{\prime}(z)\right), \quad z \in \mathbb{D} .
$$

Concerning the spectrum of $\mathrm{C}$ it is known that

$$
\sigma(\mathrm{C} ; H(\mathbb{D}))=\sigma_{p t}(\mathrm{C} ; H(\mathbb{D}))=\Sigma
$$

and that each eigenvalue $\frac{1}{n}$, for $n \in \mathbb{N}$, is simple with corresponding eigenvector $z \mapsto \frac{z^{n-1}}{(1-z)^{n}}$; see pp.71-72 of [9] with $g(z):=-\log (1-z)$ which corresponds to the Cesàro operator (1.1). In particular, $\mathrm{C}$ is always injective. Furthermore, it is also known, [5, Proposition 2.20], that

$$
\sigma^{*}(\mathrm{C} ; H(\mathbb{D}))=\overline{\sigma(\mathrm{C} ; H(\mathbb{D}))}=\Sigma_{0} \text {. }
$$


Since the spaces $A_{+}^{-\gamma}$ for $0 \leq \gamma<\infty$ and $A_{-}^{-\gamma}$ for $0<\gamma \leq \infty$ are continuously included in $H(\mathbb{D})$, we have

$$
\sigma_{p t}\left(\mathrm{C} ; A_{+}^{-\gamma}\right) \subseteq \sigma_{p t}(\mathrm{C} ; H(\mathbb{D}))=\Sigma \quad \text { and } \quad \sigma_{p t}\left(\mathrm{C} ; A_{-}^{-\gamma}\right) \subseteq \sigma_{p t}(\mathrm{C} ; H(\mathbb{D}))=\Sigma .
$$

For each $\varphi \in H^{\infty}(\mathbb{D})$ the operator $M_{\varphi}$ also belongs to $\mathcal{L}\left(A_{+}^{-\gamma}\right)$ for $0 \leq \gamma<\infty$ and to $\mathcal{L}\left(A_{-}^{-\gamma}\right)$ for $0<\gamma \leq \infty$. Since $\Sigma$ is not a compact subset of $\mathbb{C}$ the argument used for proving Proposition 1.4 also shows that $\mathrm{C} \in \mathcal{L}(H(\mathbb{D}))$ is not a compact operator.

Concerning the point $\lambda=0$ we have the following fact.

Proposition 2.2. Let $0 \leq \gamma<\infty$. Then $0 \in \sigma\left(\mathrm{C} ; A_{+}^{-\gamma}\right)$.

Proof. Suppose first that $\gamma>0$. Define $g_{0} \in H(\mathbb{D})$ by $g_{0}(z):=\frac{1}{(1+z)^{\gamma}}$, for $z \in \mathbb{D}$. Observe, for each $z \in \mathbb{D}$, that $1=|1+z-z| \leq|1+z|+|z|$ and so $\frac{(1-|z|)^{\gamma}}{|1+z|^{\gamma}} \leq 1$. Thus, $g_{0} \in A^{-\gamma} \subseteq A_{+}^{-\gamma}$ which implies that also $(1-z) g_{0}=$ $M_{1-z} g_{0} \in A^{-\gamma} \subseteq A_{+}^{-\gamma}$. Suppose that $\mathrm{C}^{-1} g_{0} \in A_{+}^{-\gamma}$. Since $M_{1-z} g_{0} \in A_{+}^{-\gamma}$, it follows from $(2.2)$ that $z(1-z) g_{0}^{\prime}=-\gamma z(1-z) \frac{1}{(1+z)^{\gamma+1}} \in A_{+}^{-\gamma}$. In particular, for $k=2$ it would follow that $\left\|z(1-z) g_{0}^{\prime}\right\|_{2}=\sup _{z \in \mathbb{D}}(1-|z|)^{\gamma+\frac{1}{2}} \mid z(1-$ $z) \mid \frac{1}{|1+z|^{\gamma+1}}<\infty$. But, each point $z_{m}:=-1+\frac{1}{m} \in \mathbb{D}$, for $m \in \mathbb{N}$ with $m \geq 2$, satisfies

$$
\begin{aligned}
& \left(1-\left|z_{m}\right|\right)^{\gamma+\frac{1}{2}}\left|z_{m}\left(1-z_{m}\right)\right| \frac{1}{\left|1+z_{m}\right|^{\gamma+1}}= \\
& \left(\frac{1}{m}\right)^{\gamma+\frac{1}{2}}\left(1-\frac{1}{m}\right)\left(2-\frac{1}{m}\right) m^{\gamma+1} \geq \frac{1}{2} m^{\frac{1}{2}}
\end{aligned}
$$

with $\frac{1}{2} m^{\frac{1}{2}} \rightarrow \infty$ for $m \rightarrow \infty$; contradiction. Thus, $\mathrm{C}$ is not surjective on $A_{+}^{-\gamma}$ and so $0 \in \sigma\left(\mathrm{C} ; A_{+}^{-\gamma}\right)$.

For $\gamma=0$, consider $g_{0}(z):=\log (1+z)=\sum_{n=1}^{\infty}(-1)^{n+1} \frac{z^{n}}{n}, z \in \mathbb{D}$. Since $\left|g_{0}(z)\right| \leq-\log (1-|z|)$ for each $z \in \mathbb{D}$, we have $g_{0} \in A_{+}^{-0}$. Suppose that $\mathrm{C}^{-1} g_{0} \in A_{+}^{-0}$. Then $z(1-z) g_{0}^{\prime}=z(1-z) \frac{1}{(1+z)} \in A_{+}^{-0}$. In particular, for $k=2$ we would have $\left\|z(1-z) g_{0}^{\prime}\right\|_{2}:=\sup _{z \in \mathbb{D}}(1-|z|)^{\frac{1}{2}}|z(1-z)| \frac{1}{|1+z|}<\infty$. But, each point $z_{m}:=-1+\frac{1}{m} \in \mathbb{D}$, for $m \in \mathbb{N}$ with $m \geq 2$, satisfies

$$
\left(1-\left|z_{m}\right|\right)^{\frac{1}{2}}\left|z_{m}\left(1-z_{m}\right)\right| \frac{1}{\left|1+z_{m}\right|}=\left(\frac{1}{m}\right)^{\frac{1}{2}}\left(1-\frac{1}{m}\right)\left(2-\frac{1}{m}\right) m \geq \frac{1}{2} m^{\frac{1}{2}},
$$

which is again a contradiction. So, $0 \in \sigma\left(\mathrm{C} ; A_{+}^{-0}\right)$ also in this case.

Proposition 2.3. Let $0 \leq \gamma<\infty$. Then

$$
\sigma\left(\mathrm{C} ; A_{+}^{-\gamma}\right)=\{0\} \cup\left\{\frac{1}{m}: m \in \mathbb{N}, m \leq \gamma\right\} \cup\left\{\lambda \in \mathbb{C}:\left|\lambda-\frac{1}{2 \gamma}\right|<\frac{1}{2 \gamma}\right\} .
$$

For $\gamma=0$ we have

$$
\sigma\left(\mathrm{C} ; A_{+}^{-0}\right)=\{0\} \cup\{\lambda \in \mathbb{C}: \operatorname{Re}(\lambda)>0\} .
$$


Proof. Propositions 2.1 and 2.2 imply that

$$
\{0\} \cup\left\{\frac{1}{m}: m \in \mathbb{N}, m \leq \gamma\right\} \subseteq \sigma\left(\mathrm{C} ; A_{+}^{-\gamma}\right), \quad 0 \leq \gamma<\infty .
$$

Moreover, Lemma 5.1 in the Appendix, applied to $T:=\mathrm{C}$ acting on $A_{+}^{-\gamma}$ and to $T_{n}:=\mathrm{C}_{n}=\mathrm{C}$ acting on $A_{0}^{-\left(\gamma+\frac{1}{n}\right)}$, implies that

$$
\sigma\left(\mathrm{C} ; A_{+}^{-\gamma}\right) \subseteq \cup_{n \in \mathbb{N}} \sigma\left(\mathrm{C}_{n} ; A_{0}^{-\left(\gamma+\frac{1}{n}\right)}\right) .
$$

By Theorem 1.1(i)-(ii) we have, for each $\gamma>0$, that

$$
\cup_{n \in \mathbb{N}} \sigma\left(\mathrm{C}_{n} ; A_{0}^{-\left(\gamma+\frac{1}{n}\right)}\right) \subseteq\{0\} \cup\left\{\frac{1}{m}: m \in \mathbb{N}, m \leq \gamma\right\} \cup\left\{\lambda \in \mathbb{C}:\left|\lambda-\frac{1}{2 \gamma}\right|<\frac{1}{2 \gamma}\right\},
$$

and for $\gamma=0$ that $\sigma\left(\mathrm{C} ; A_{+}^{-0}\right) \subseteq\{0\} \cup\{\lambda \in \mathbb{C}: \operatorname{Re}(\lambda)>0\}$. So, to complete the proof it remains to show that

$$
\left\{\lambda \in \mathbb{C}:\left|\lambda-\frac{1}{2 \gamma}\right|<\frac{1}{2 \gamma}\right\} \subseteq \sigma\left(\mathrm{C} ; A_{+}^{-\gamma}\right), \gamma>0,
$$

and that

$$
\{\lambda \in \mathbb{C}: \operatorname{Re}(\lambda)>0\} \subseteq \sigma\left(\mathrm{C} ; A_{+}^{-0}\right), \gamma=0 .
$$

Fix $\gamma>0$. Let $\lambda \in \mathbb{C}$ satisfy $\left|\lambda-\frac{1}{2 \gamma}\right|<\frac{1}{2 \gamma}$ and set $m_{\gamma}:=\max \{m \in$ $\left.\mathbb{N}: \frac{1}{m} \geq \frac{1}{\gamma}\right\}$. Then there exists $n_{0} \in \mathbb{N}$ such that $\frac{1}{\gamma+n^{-1}} \geq \frac{1}{m_{\gamma}+1}$ and $\left|\lambda-\frac{1}{2\left(\gamma+\frac{1}{n}\right)}\right|<\frac{1}{2\left(\gamma+\frac{1}{n}\right)}$ for all $n \geq n_{0}$. By Theorem 1.1(iii), the operator $\lambda I-\mathrm{C}_{n}$ is injective and $\operatorname{Im}\left(\lambda I-\mathrm{C}_{n}\right)$ is a closed, one-codimensional subspace of $A_{0}^{-\left(\gamma+\frac{1}{n}\right)}$ for all $n \geq n_{0}$. We proceed to show that $\operatorname{Im}(\lambda I-\mathrm{C})$ is a proper closed subspace of $A_{+}^{-\gamma}$. The argument is as in the proof of [4, Theorem $2.2]$.

To show that $\operatorname{Im}(\lambda I-\mathrm{C})$ is a closed subspace of $A_{+}^{-\gamma}$, let $\left(g_{j}\right)_{j \in \mathbb{N}} \subseteq$ $\operatorname{Im}(\lambda I-\mathrm{C})$ be a sequence in $A_{+}^{-\gamma}$ converging to $g \in A_{+}^{-\gamma}$. For each $j \in \mathbb{N}$ select $f_{j} \in A_{+}^{-\gamma}$ such that $(\lambda I-\mathrm{C}) f_{j}=g_{j}$. In particular, $\left(f_{j}\right)_{j \in \mathbb{N}} \subseteq A_{0}^{-\left(\gamma+\frac{1}{n}\right)}$ (hence, also $\left.\left(g_{j}\right)_{j \in \mathbb{N}} \subseteq A_{0}^{-\left(\gamma+\frac{1}{n}\right)}\right)$ for all $n \geq n_{0}$. Since for each $n \geq n_{0}$ the space $A_{+}^{-\gamma} \subseteq A_{0}^{-\left(\gamma+\frac{1}{n}\right)}$ continuously, we have $g_{j} \rightarrow g$ in the Banach space $A_{0}^{-\left(\gamma+\frac{1}{n}\right)}$ as $j \rightarrow \infty$. But, $\operatorname{Im}\left(\lambda I-\mathrm{C}_{n}\right)$ is a closed subspace of $A_{0}^{-\left(\gamma+\frac{1}{n}\right)}$ and so $g \in \operatorname{Im}\left(\lambda I-\mathrm{C}_{n}\right)$ for all $n \geq n_{0}$. Thus, for each $n \geq n_{0}$ there exists $h_{n} \in A_{0}^{-\left(\gamma+\frac{1}{n}\right)}$ satisfying $\left(\lambda I-C_{n}\right) h_{n}=g$. Moreover, for $n \geq n_{0}$ we have $\left(\lambda I-\mathrm{C}_{n}\right) h_{n}=g=\left(\lambda I-\mathrm{C}_{n+1}\right) h_{n+1}$. Since the restriction of $\mathrm{C}_{n}$ to $A_{0}^{-\left(\gamma+\frac{1}{n+1}\right)}$ coincides with $\mathrm{C}_{n+1}$ and $\left(\lambda I-\mathrm{C}_{n}\right)$ is injective, it follows that $h_{n}=h_{n+1}$ for all $n \geq n_{0}$. Accordingly, $h_{n_{0}} \in A_{+}^{-\gamma}$ and $(\lambda I-\mathrm{C}) h_{n_{0}}=g$, i.e., $g \in \operatorname{Im}(\lambda I-C)$.

To see that $\operatorname{Im}(\lambda I-\mathrm{C})$ is a proper closed subspace of $A_{+}^{-\gamma}$ assume the contrary, that is, $\operatorname{Im}(\lambda I-\mathrm{C})=A_{+}^{-\gamma}$. It was already noted that $A_{+}^{-\gamma}$ is dense in $A_{0}^{-\left(\gamma+\frac{1}{n}\right)}$ for all $n \in \mathbb{N}$. This implies, for every $n \in \mathbb{N}$, that

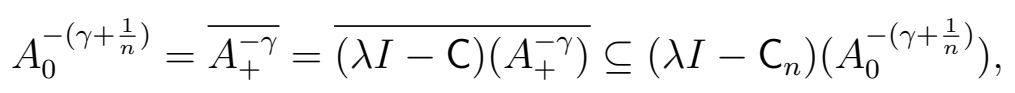


with all closures taken in $A_{0}^{-\left(\gamma+\frac{1}{n}\right)}$. This contradicts $\operatorname{Im}\left(\lambda I-\mathrm{C}_{n}\right)$ being a closed, one-codimensional subspace of $A_{0}^{-\left(\gamma+\frac{1}{n}\right)}$ for all $n \geq n_{0}$. So, $(\lambda I-\mathrm{C})$ is not surjective, i.e., $\lambda \in \sigma\left(\mathrm{C} ; A_{+}^{-\gamma}\right)$.

Consider now $\gamma=0$ in which case $A_{0}^{-0}=\cap_{\mu>0} A_{0}^{-\mu}$. Let $\lambda$ satisfy $\operatorname{Re}(\lambda)>$ 0 and choose $\gamma_{0} \in(0,1)$ such that $\left|\lambda-\frac{1}{2 \gamma_{0}}\right|<\frac{1}{2 \gamma_{0}}$. Then also $\left|\lambda-\frac{1}{2 \gamma}\right|<\frac{1}{2 \gamma}$ for all $0<\gamma \leq \gamma_{0}$. Observe that

$$
\Sigma \subseteq\left\{z \in \mathbb{C}:\left|z-\frac{1}{2 \gamma_{0}}\right|<\frac{1}{2 \gamma_{0}}\right\} \subseteq\left\{z \in \mathbb{C}:\left|z-\frac{1}{2 \gamma}\right|<\frac{1}{2 \gamma}\right\}
$$

and $\left\{\frac{1}{m}: m \in \mathbb{N}, m \leq \gamma\right\}=\emptyset$ for all $0<\gamma \leq \gamma_{0}$. In particular, Theorem 1.1(i),(iii) shows that $\sigma_{p t}\left(\mathrm{C} ; A_{0}^{-\gamma}\right)=\emptyset$, i.e., $\lambda I-\mathrm{C}$ is injective on $A_{0}^{-\gamma}$, and that $\operatorname{Im}(\lambda I-\mathrm{C})$ is a closed, one-codimensional subspace of $A_{0}^{-\gamma}$ for all $0<\gamma \leq \gamma_{0}$. The proof now continues as above (for the case of $\gamma>0$ ) to show that $\operatorname{Im}(\lambda I-\mathrm{C})$ is a proper closed subspace of $A_{+}^{-0}$ and hence, $\lambda \in \sigma\left(\mathrm{C} ; A_{+}^{-0}\right)$.

Corollary 2.4. Let $0 \leq \gamma<\infty$. Then $\sigma^{*}\left(\mathrm{C} ; A_{+}^{-\gamma}\right)=\overline{\sigma\left(\mathrm{C} ; A_{+}^{-\gamma}\right)}$.

Proof. This is a direct consequence of Proposition 2.3 and Lemma 5.1 (in the Appendix $)$, since $\cup_{n \in \mathbb{N}} \sigma\left(\mathrm{C}_{n} ; A_{0}^{-\left(\gamma+\frac{1}{n}\right)}\right) \subseteq \overline{\sigma\left(\mathrm{C} ; A_{+}^{-\gamma}\right)}$; this inclusion was established in the proof of Proposition 2.3.

Propositions 2.1-2.3 and Corollary 2.4 together provide the complete proof of Theorem 1.2.

The proof of Theorem 1.3 is also a consequence of several propositions.

Proposition 2.5. Let $0<\gamma \leq \infty$. Then

$$
\sigma_{p t}\left(\mathrm{C} ; A_{-}^{-\gamma}\right)=\left\{\frac{1}{m}: m \in \mathbb{N}, m<\gamma\right\} .
$$

Proof. Applying Lemma 5.2(i) of the Appendix to $T:=\mathrm{C}$ acting in $A_{-}^{-\gamma}$ and $T_{n}:=\mathrm{C}_{n}=\mathrm{C}$ acting in $A_{0}^{-\left(\gamma-\frac{1}{n}\right)}$ together with Theorem 1.1(i), we obtain that

$$
\begin{aligned}
\sigma_{p t}\left(\mathrm{C} ; A_{-}^{-\gamma}\right) & =\cup_{n \in \mathbb{N}} \sigma_{p t}\left(\mathrm{C}_{n} ; A_{0}^{-\left(\gamma-\frac{1}{n}\right)}\right)=\cup_{n \in \mathbb{N}}\left\{\frac{1}{m}: m \in \mathbb{N}, m<\left(\gamma-\frac{1}{n}\right)\right\} \\
& =\left\{\frac{1}{m}: m \in \mathbb{N}, m<\gamma\right\} .
\end{aligned}
$$

The case of the Korenblum space, i.e., $\gamma=\infty$, follows similarly.

Proposition 2.6. Let $0<\gamma<\infty$. Then

$$
\sigma\left(\mathrm{C} ; A_{-}^{-\gamma}\right) \subseteq\left\{\frac{1}{m}: m \in \mathbb{N}, m<\gamma\right\} \cup\left\{\lambda \in \mathbb{C}:\left|\lambda-\frac{1}{2 \gamma}\right| \leq \frac{1}{2 \gamma}\right\} .
$$

Proof. Applying Lemma 5.2(ii) of the Appendix to $T:=\mathrm{C}$ acting in $A_{-}^{-\gamma}$ and $T_{n}:=\mathrm{C}_{n}=\mathrm{C}$ acting in $A_{0}^{-\left(\gamma-\frac{1}{n}\right)}$ we obtain that

$$
\sigma\left(\mathrm{C} ; A_{-}^{-\gamma}\right) \subseteq \cap_{m \in \mathbb{N}, m>\gamma^{-1}}\left(\cup_{n=m}^{\infty} \sigma\left(\mathrm{C}_{n} ; A_{0}^{-\left(\gamma-\frac{1}{n}\right)}\right)\right) .
$$


On the other hand, Theorem 1.1(ii) implies, for every $n \in \mathbb{N}$ with $n>\gamma^{-1}$, that

$\sigma\left(\mathrm{C} ; A^{-\left(\gamma-\frac{1}{n}\right)}\right)=\left\{\frac{1}{m}: m \in \mathbb{N}, m<\left(\gamma-\frac{1}{n}\right)\right\} \cup\left\{\lambda \in \mathbb{C}:\left|\lambda-\frac{1}{2\left(\gamma-\frac{1}{n}\right)}\right| \leq \frac{1}{2\left(\gamma-\frac{1}{n}\right)}\right\}$.

Since, for each $m, n \in \mathbb{N}$ satisfying $n \geq m>\gamma^{-1}$, we have

$$
\left\{\lambda \in \mathbb{C}:\left|\lambda-\frac{1}{2\left(\gamma-\frac{1}{n}\right)}\right| \leq \frac{1}{2\left(\gamma-\frac{1}{n}\right)}\right\} \subseteq\left\{\lambda \in \mathbb{C}:\left|\lambda-\frac{1}{2\left(\gamma-\frac{1}{m}\right)}\right| \leq \frac{1}{2\left(\gamma-\frac{1}{m}\right)}\right\},
$$

it follows that

$$
\sigma\left(\mathrm{C} ; A_{-}^{-\gamma}\right) \subseteq\left\{\frac{1}{m}: m \in \mathbb{N}, m<\gamma\right\} \cup\left\{\lambda \in \mathbb{C}:\left|\lambda-\frac{1}{2 \gamma}\right| \leq \frac{1}{2 \gamma}\right\} .
$$

Proposition 2.7. Let $0<\gamma<\infty$. Then

$$
\left\{\frac{1}{m}: m \in \mathbb{N}, m<\gamma\right\} \cup\left\{\lambda \in \mathbb{C}:\left|\lambda-\frac{1}{2 \gamma}\right|<\frac{1}{2 \gamma}\right\} \subseteq \sigma\left(\mathrm{C} ; A_{-}^{-\gamma}\right) .
$$

Proof. First, Proposition 2.5 implies $\left\{\frac{1}{m}: m \in \mathbb{N}, m<\gamma\right\} \subseteq \sigma\left(\mathrm{C} ; A_{-}^{-\gamma}\right)$.

Assume there exists $\lambda \in \mathbb{C}$ satisfying $\left|\lambda-\frac{1}{2 \gamma}\right|<\frac{1}{2 \gamma}$ but, $\lambda \notin \sigma\left(\mathrm{C} ; A_{-}^{-\gamma}\right)$. Then $(\lambda I-\mathrm{C})\left(A_{-}^{-\gamma}\right)=A_{-}^{-\gamma}$. On the other hand, Theorem 1.1(iii) yields that $(\lambda I-\mathrm{C})\left(A_{0}^{-\gamma}\right)$ is a closed, one-codimensional subspace of $A_{0}^{-\gamma}$. The density of $A_{-}^{-\gamma}$ in $A_{0}^{-\gamma}$ then implies that

$$
A_{0}^{-\gamma}=\overline{A_{-}^{-\gamma}}=\overline{(\lambda I-\mathrm{C})\left(A_{-}^{-\gamma}\right)} \subseteq(\lambda I-\mathrm{C})\left(A_{0}^{-\gamma}\right),
$$

with all closures taken in $A_{0}^{-\gamma}$. Hence, $(\lambda I-\mathrm{C})\left(A_{0}^{-\gamma}\right)=A_{0}^{-\gamma}$; contradiction.

Proposition 2.8. Let $0<\gamma<\infty$. Then

$$
\sigma\left(\mathrm{C} ; A_{-}^{-\gamma}\right)=\left\{\frac{1}{m}: m \in \mathbb{N}, m<\gamma\right\} \cup\left\{\lambda \in \mathbb{C}:\left|\lambda-\frac{1}{2 \gamma}\right| \leq \frac{1}{2 \gamma}\right\} .
$$

Proof. By Propositions 2.6 and 2.7 it remains to establish that

$$
\left\{\lambda \in \mathbb{C}:\left|\lambda-\frac{1}{2 \gamma}\right|=\frac{1}{2 \gamma}\right\} \subseteq \sigma\left(\mathrm{C} ; A_{-}^{-\gamma}\right) .
$$

So, fix $\lambda \in \mathbb{C}$ such that $\left|\lambda-\frac{1}{2 \gamma}\right|=\frac{1}{2 \gamma}$, in which case $\operatorname{Re}\left(\frac{1}{\lambda}\right)=\gamma$. By $[31$, Proposition 4], the constant function $\mathbf{1} \notin(\lambda I-\mathrm{C})\left(A_{0}^{-\gamma}\right)$. Since $\mathbf{1} \in A_{-}^{-\gamma}$ and $A_{-}^{-\gamma} \subseteq A_{0}^{-\gamma}$, it follows that $(\lambda I-\mathrm{C}): A_{-}^{-\gamma} \rightarrow A_{-}^{-\gamma}$ is not surjective, i.e., $\lambda \in \sigma\left(\mathrm{C} ; A_{-}^{-\gamma}\right)$.

Proposition 2.9. Let $0<\gamma<\infty$. Then

$$
\sigma^{*}\left(\mathrm{C} ; A_{-}^{-\gamma}\right)=\sigma\left(\mathrm{C} ; A_{-}^{-\gamma}\right) .
$$

Proof. It is always the case that $\sigma\left(\mathrm{C} ; A_{-}^{-\gamma}\right) \subseteq \sigma^{*}\left(\mathrm{C} ; A_{-}^{-\gamma}\right)$; see Section 1 .

To establish the reverse inclusion, fix $\lambda \notin \sigma\left(\mathrm{C} ; A_{-}^{-\gamma}\right)$. By Proposition 2.8 the set $\sigma\left(\mathrm{C} ; A_{-}^{-\gamma}\right)$ is compact and so there exist $r>0$ and $n_{0} \in \mathbb{N}$ with $n_{0}>\frac{1}{\gamma}$ such that

$$
\overline{B(\lambda, r)} \cap\left(\left\{\frac{1}{m}: m \in \mathbb{N}, m<\gamma\right\} \cup\left\{\lambda \in \mathbb{C}:\left|\lambda-\frac{1}{2\left(\gamma-\frac{1}{n_{0}}\right)}\right| \leq \frac{1}{2\left(\gamma-\frac{1}{n_{0}}\right)}\right\}\right)=\emptyset .
$$


Applying Theorem 1.1(ii) it follows that $\overline{B(\lambda, r)} \cap \sigma\left(\mathrm{C}_{n} ; A_{0}^{-\left(\gamma-\frac{1}{n}\right)}\right)=\emptyset$, for every $n \geq n_{0}$, that is,

$$
\overline{B(\lambda, r)} \subseteq \rho\left(\mathrm{C}_{n} ; A_{0}^{-\left(\gamma-\frac{1}{n}\right)}\right), \quad n \geq n_{0},
$$

where $\mathrm{C}_{n}$ is the restriction of $\mathrm{C}$ to $A_{0}^{-\left(\gamma-\frac{1}{n}\right)}$. Therefore, the set $\left\{R\left(\mu, \mathrm{C}_{n}\right): \mu \in\right.$ $\overline{B(\lambda, r)}\}$ is equicontinuous, that is, operator norm bounded in $\mathcal{L}\left(A_{0}^{-\left(\gamma-\frac{1}{n}\right)}\right)$ for every $n \geq n_{0}$; here, $R\left(\mu, \mathrm{C}_{n}\right)$ denotes the inverse of the bijective operator $\left(\mu I-\mathrm{C}_{n}\right): A_{0}^{-\left(\gamma-\frac{1}{n}\right)} \rightarrow A_{0}^{-\left(\gamma-\frac{1}{n}\right)}$ for $\mu \in \overline{B(\lambda, r)}$. The claim is that the set $\{R(\mu, \mathrm{C}): \mu \in \overline{B(\lambda, r)}\}$ is equicontinuous in $\mathcal{L}\left(A_{-}^{-\gamma}\right)$, where now $R(\mu, \mathrm{C})$ denotes the inverse of the bijective operator $(\mu I-\mathrm{C}): A_{-}^{-\gamma} \rightarrow A_{-}^{-\gamma}$ for $\mu \in \overline{B(\lambda, r)}$. This would verify that $\lambda \notin \sigma^{*}\left(\mathrm{C} ; A_{-}^{-\gamma}\right)$.

To establish the claim it suffices to show that $\{R(\mu, \mathrm{C}) f: \mu \in \overline{B(\lambda, r)}\}$ is a bounded set in $A_{-}^{-\gamma}$ for all $f \in A_{-}^{-\gamma}$. This is because $A_{-}^{-\gamma}$ is a (DFS)-space (hence, barrelled) and so the Banach-Steinhaus principle is applicable. So, fix $f \in A_{-}^{-\gamma}$. Then $f \in A_{0}^{-\left(\gamma-\frac{1}{n}\right)}$ for some $n \geq n_{0}$. So, $\left\{R\left(\mu, \mathrm{C}_{n}\right) f: \mu \in\right.$ $\overline{B(\lambda, r)}\}$ is a bounded set in $A_{0}^{-\left(\gamma-\frac{1}{n}\right)}$ and hence, also in $A_{-}^{-\gamma}$. Since the restriction $\left.R(\mu, \mathrm{C})\right|_{A_{0}^{-\left(\gamma-\frac{1}{n}\right)}}=R\left(\mu, \mathrm{C}_{n}\right)$ for $\mu \in \overline{B(\lambda, r)}$, the proof is complete.

It remains to complete the proof of part (b) of Theorem 1.3 for $\mathrm{C}$ acting in the Korenblum space $A^{-\infty}=A_{-}^{-\infty}$.

To this effect, observe that Proposition 2.5, with $\gamma=\infty$, shows that $\sigma_{p t}\left(\mathrm{C} ; A^{-\infty}\right)=\Sigma$. Suppose that $\lambda \in \mathbb{C} \backslash \Sigma_{0}$. Then there exists $n(0) \in \mathbb{N}$ such that $\lambda \notin \Sigma \cup\left\{z \in \mathbb{C}:\left|z-\frac{1}{2 n(0)}\right| \leq \frac{1}{n(0)}\right\}$. Therefore $\lambda \notin \sigma\left(\mathrm{C}_{n} ; A^{-n}\right)$ for each $n \geq n(0)$ where $\mathrm{C}_{n}:=\left.\mathrm{C}\right|_{A^{-n}}$; see Theorem 1.1(i),(ii). By Lemma 5.2 (ii) in the Appendix we can conclude that $\lambda \notin \sigma\left(\mathrm{C} ; A^{-\infty}\right)$. Hence, we have established that

$$
\Sigma=\sigma_{p t}\left(\mathrm{C} ; A^{-\infty}\right) \subseteq \sigma\left(\mathrm{C} ; A^{-\infty}\right) \subseteq \Sigma_{0} .
$$

Next we prove that $0 \notin \sigma\left(\mathrm{C} ; A^{-\infty}\right)$. As observed above (see $\left.(2.2)\right), \mathrm{C}^{-1} \in$ $\mathcal{L}(H(\mathbb{D}))$ is given by $\mathrm{C}^{-1}=M_{1-z} D M_{z}$. Clearly both $M_{z}, M_{1-z}$ belong to $\mathcal{L}\left(A^{-\infty}\right)$. Actually, also $D \in \mathcal{L}\left(A^{-\infty}\right)$, [30, Remark 1]. Accordingly, $\mathrm{C}^{-1}=M_{1-z} D M_{z} \in \mathcal{L}\left(A^{-\infty}\right)$, that is, $0 \in \rho\left(\mathrm{C} ; A^{-\infty}\right)$. So, we have verified that $\sigma\left(\mathrm{C} ; A^{-\infty}\right)=\sigma_{p t}\left(\mathrm{C} ; A^{-\infty}\right)=\Sigma$, which is precisely part (i) of Theorem $1.3(\mathrm{~b})$.

The inclusion $\overline{\sigma\left(\mathrm{C} ; A^{-\infty}\right)} \subseteq \sigma^{*}\left(\mathrm{C} ; A^{-\infty}\right)$ holds in general. Suppose that $\lambda \notin \overline{\sigma\left(\mathrm{C} ; A^{-\infty}\right)}=\Sigma_{0}$; see (2.3). By the argument leading to (2.3), there exist $n(0) \in \mathbb{N}$ and $r>0$ such that $\overline{B(\lambda, r)} \cap \sigma\left(\mathrm{C}_{n} ; A^{-n}\right)=\emptyset$ for each $n \geq n(0)$, where $\mathrm{C}_{n}:=\mathrm{C}_{A_{0}^{-n}}$. Moreover, the set $\left\{R\left(\mu, \mathrm{C}_{n}\right): \mu \in \overline{B(\lambda, r)}\right\}$ is equicontinuous, that is, operator norm bounded in $\mathcal{L}\left(A_{0}^{-n}\right)$ for every 
$n \geq n(0)$, where $R\left(\mu, \mathrm{C}_{n}\right)$ denotes the inverse of the bijective operator $\left(\mu I-\mathrm{C}_{n}\right): A_{0}^{-n} \rightarrow A_{0}^{-n}$ for $\mu \in \overline{B(\lambda, r)}$. The proof that $\{R(\mu, \mathrm{C}): \mu \in$ $\overline{B(\lambda, r)}\}$ is equicontinuous in $\mathcal{L}\left(A^{-\infty}\right)$ is now similar to that in the proof of Proposition 2.9; here, $R(\mu, \mathrm{C})$ denotes the inverse of the bijective operator $(\mu I-\mathrm{C}): A^{-\infty} \rightarrow A^{-\infty}$ for $\mu \in \overline{B(\lambda, r)}$. Accordingly, $\lambda \notin \sigma^{*}\left(\mathrm{C} ; A^{-\infty}\right)$. This shows that $\sigma^{*}\left(\mathrm{C} ; A^{-\infty}\right) \subseteq \overline{\sigma\left(\mathrm{C} ; A^{-\infty}\right)}$. The proof of part (ii) of Theorem $1.3(\mathrm{~b})$ is thereby complete.

We end this section with the following application of the main results.

Proposition 2.10. The differentiation operator $D$ belongs to $\mathcal{L}\left(A^{-\infty}\right)$ and

$$
\sigma\left(D ; A^{-\infty}\right)=\sigma_{p t}\left(D ; A^{-\infty}\right)=\mathbb{C} .
$$

The operator $D$ fails to act in $A_{+}^{-\gamma}$ for every $0 \leq \gamma<\infty$ and in $A_{-}^{-\gamma}$ for every $0<\gamma<\infty$.

Proof. We already observed earlier that $D \in \mathcal{L}\left(A^{-\infty}\right)$. Since $f_{\lambda}(z):=e^{\lambda z}$, for $z \in \mathbb{D}$, belongs to $A^{-\infty}$ for every $\lambda \in \mathbb{C}$ and satisfies $D f_{\lambda}=\lambda f_{\lambda}$, it is immediate that (2.4) holds.

Fix $0 \leq \gamma<\infty$ and suppose that $D \in \mathcal{L}\left(A_{+}^{-\gamma}\right)$. Since both $M_{z}, M_{1-z} \in$ $\mathcal{L}\left(A_{+}^{-\gamma}\right)$, it would follow that $M_{1-z} D M_{z} \in \mathcal{L}\left(A_{+}^{-\gamma}\right)$. But, $M_{1-z} D M_{z}=$ $C^{-1}$ holds in $H(\mathbb{D})$ and hence, this identity is also valid in $\mathcal{L}\left(A_{+}^{-\gamma}\right)$, i.e., $0 \in \rho\left(\mathrm{C} ; A_{+}^{-\gamma}\right)$. This contradicts parts (a)(ii) and (b)(ii) of Theorem 1.2. Accordingly, $D$ does not act in $A_{+}^{-\gamma}$.

For each $0<\gamma<\infty$, a similar argument can be used to show (via Theorem 1.3(a)(ii)) that $D$ also fails to act in $A_{-}^{-\gamma}$.

\section{MEAN ERGODICITY.}

An operator $T \in \mathcal{L}(X)$, with $X$ a lcHs, is power bounded if $\left\{T^{n}\right\}_{n=1}^{\infty}$ is an equicontinuous subset of $\mathcal{L}(X)$. For a Banach space $X$, this means that $\sup _{n \in \mathbb{N}}\left\|T^{n}\right\|_{o p}<\infty$. Given $T \in \mathcal{L}(X)$, the averages

$$
T_{[n]}:=\frac{1}{n} \sum_{m=1}^{n} T^{m}, \quad n \in \mathbb{N},
$$

are called the Cesàro means of $T$. The operator $T$ is said to be mean ergodic (resp., uniformly mean ergodic) if $\left\{T_{[n]}\right\}_{n=1}^{\infty}$ is a convergent sequence in $\mathcal{L}_{s}(X)$ (resp., in $\mathcal{L}_{b}(X)$ ). It follows from $(3.1)$ that $\frac{T^{n}}{n}=T_{[n]}-\frac{n-1}{n} T_{[n-1]}$, for $n \geq 2$. Hence, $\tau_{s}-\lim _{n \rightarrow \infty} \frac{T^{n}}{n}=0$ whenever $T$ is mean ergodic. A relevant text for mean ergodic operators is [27]; see also [1, 21]. Every power bounded operator on a Montel Fréchet space or an (LF)-space which is Montel is necessarily uniformly mean ergodic, [1, Proposition 2.8].

The Fréchet space $H(\mathbb{D})$ is isomorphic to the power series space $\Lambda_{0}(\alpha)$ of finite type (for the weight $\alpha:=(n)_{n \in \mathbb{N}}$ ). This isomorphism, specified by sending $f \in H(\mathbb{D})$ to the sequence of its Taylor coefficients, transforms $\mathrm{C} \in$ $\mathcal{L}(H(\mathbb{D}))$ into the discrete Cesàro operator acting in the Fréchet space $\mathbb{C}^{\mathbb{N}_{0}}$; 
see (1.2). Accordingly, the following result is a straight-forward consequence of Propositions 3.1-3.3 in [5].

Proposition 3.1. The Cesàro operator $\mathrm{C} \in \mathcal{L}(H(\mathbb{D}))$ is power bounded and uniformly mean ergodic. In particular, $\operatorname{Im}(I-\mathrm{C})$ is a closed subspace of $H(\mathbb{D})$ and

$$
H(\mathbb{D})=\operatorname{Ker}(I-\mathrm{C}) \oplus \operatorname{Im}(I-\mathrm{C}) .
$$

Actually, $\operatorname{Im}(I-\mathrm{C})^{m}$ is a closed subspace of $H(\mathbb{D})$ for all $m \in \mathbb{N}$. Moreover, the sequence of iterates $\left\{\mathrm{C}^{m}\right\}_{m \in \mathbb{N}}$ is convergent in $\mathcal{L}_{b}(H(\mathbb{D}))$.

The following result will be needed for later proofs.

Lemma 3.2. $\quad$ (i) The Cesàro operator $\mathrm{C} \in \mathcal{L}(H(\mathbb{D}))$ satisfies both

$$
\operatorname{Ker}(I-\mathrm{C})=\operatorname{span}\{1 /(1-z)\}
$$

and

$$
\overline{\operatorname{Im}(I-\mathrm{C})}=\operatorname{Im}(I-\mathrm{C})=\{h \in H(\mathbb{D}): h(0)=0\} .
$$

(ii) Let $X$ be a barrelled lcHs of analytic functions on $\mathbb{D}$ which contains the constant functions, is continuously included in $H(\mathbb{D})$ and such that $\mathrm{C}: X \rightarrow X$ is continuous. If $\mathrm{C}: X \rightarrow X$ is mean ergodic, then $1 /(1-z) \in X$.

Proof. (i) This is proved by Persson in [31, Section 2]; see (2.4) and (2.5) on p.1184 with $\lambda=1$.

(ii) Assume that C $: X \rightarrow X$ is continuous and mean ergodic. Then

$$
X=\operatorname{Ker}\left(I-\mathrm{C}_{X}\right) \oplus \overline{\operatorname{Im}\left(I-\mathrm{C}_{X}\right)} \text {, }
$$

where $C_{X}$ denotes the restriction of $C$ to $X$ and the closure of $\operatorname{Im}\left(I-C_{X}\right)$ is formed in $X$, [1, Theorem 2.4]. If $1 /(1-z) \notin X$, then $\operatorname{Ker}\left(I-\mathrm{C}_{X}\right)=\{0\}$ by part (i). Since $\overline{\operatorname{Im}\left(I-\mathrm{C}_{X}\right)} X \subseteq \overline{\operatorname{Im}(I-\mathrm{C})}$, it follows from part (i) and (3.2) that $X \subseteq\{h \in H(\mathbb{D}): h(0)=0\}$. This is a contradiction, since $\mathbf{1} \in X$.

We first treat $\mathrm{C}$ when it acts in the Fréchet spaces $A_{+}^{-\gamma}$ for $0 \leq \gamma<\infty$.

Proposition 3.3. $\quad$ (i) Let $1 \leq \gamma<\infty$. Then $\mathrm{C} \in \mathcal{L}\left(A_{+}^{-\gamma}\right)$ is both power bounded and uniformly mean ergodic.

(ii) Let $0 \leq \gamma<1$. Then $\mathrm{C} \in \mathcal{L}\left(A_{+}^{-\gamma}\right)$ is neither power bounded nor mean ergodic.

Proof. (i) Fix $\gamma \in[1, \infty)$. Then $A_{+}^{-\gamma}=\cap_{k=1}^{\infty} A^{-\left(\gamma+\frac{1}{k}\right)}$. By Theorem 2.3(i) of [3], $\mathrm{C} \in \mathcal{L}\left(A^{-\left(\gamma+\frac{1}{k}\right)}\right)$ has operator norm equal to 1 in each Banach space $A^{-\left(\gamma+\frac{1}{k}\right)}, k \in \mathbb{N}$. From the definition of the norms $\|\cdot\|_{k}$, for $k \in \mathbb{N}$, which determine the topology of the Fréchet space $A_{+}^{-\gamma}$ it is routine to verify that $\mathrm{C} \in \mathcal{L}\left(A_{+}^{-\gamma}\right)$ is power bounded. Since $A_{+}^{-\gamma}$ is Montel, we can apply [1, Proposition 2.8] to conclude that $\mathrm{C}$ is uniformly mean ergodic.

(ii) Suppose now that $0 \leq \gamma<1$. Since $A_{+}^{-\gamma}$ is Montel, if $\mathrm{C}$ is power bounded on $A_{+}^{-\gamma}$, then it is necessarily (even uniformly) mean ergodic, [1, 
Proposition 2.8]. Then, by Lemma 3.2(ii), the function $1 /(1-z) \in A_{+}^{-\gamma}$ and hence, $1 /(1-z) \in A_{0}^{-1}$ because $\gamma<1$. This is impossible as $\frac{1-|z|}{|1-z|}=1$ for all real $z \in[0,1)$.

We now turn to the (DFS)-spaces $A_{-}^{-\gamma}$ for $0<\gamma \leq \infty$.

Proposition 3.4. (i) Let $1<\gamma \leq \infty$. Then $\mathrm{C} \in \mathcal{L}\left(A_{-}^{-\gamma}\right)$ is both power bounded and uniformly mean ergodic.

(ii) Let $0<\gamma \leq 1$. Then $\mathrm{C} \in \mathcal{L}\left(A_{-}^{-\gamma}\right)$ is neither power bounded nor mean ergodic.

Proof. (i) Fix $1<\gamma \leq \infty$. Then $A_{-}^{-\gamma}$ is the increasing union of the Banach spaces $A^{-\mu}$, for $1<\mu<\gamma$. Moreover, $\mathrm{C}$ has operator norm equal to 1 on each space $A^{-\mu}$, for $1<\mu<\gamma$, [3, Theorem 2.3(i)]. Fix $f \in A_{-}^{-\gamma}$. Then the orbit $\left\{\mathrm{C}^{m} f: m \in \mathbb{N}\right\}$ of $f$ is contained and bounded in one of Banach space steps of the inductive limit. Hence, $\left\{\mathrm{C}^{m}: m \in \mathbb{N}\right\}$ is a bounded set in $\mathcal{L}_{s}\left(A_{-}^{-\gamma}\right)$ and so, by the Banach Steinhaus principle, it is necessarily equicontinuous in $\mathcal{L}\left(A_{-}^{-\gamma}\right)$ as the space $A_{-}^{-\gamma}$ is barrelled. Since the space $A_{-}^{-\gamma}$ is Montel, Proposition 2.8 of [1] implies that $\mathrm{C}$ is uniformly mean ergodic.

(ii) Fix $\gamma \in(0,1]$. If $\mathrm{C}$ is power bounded on $A_{-}^{-\gamma}$, then it is (uniformly) mean ergodic because $A_{-}^{-\gamma}$ is a Montel space, [1, Proposition 2.8]. Then Lemma 3.2(ii) implies that $1 /(1-z) \in A_{-}^{-\gamma}$. By the definition of $A_{-}^{-\gamma}$ there exists $\mu<\gamma \leq 1$ such that $1 /(1-z) \in A^{-\mu}$. This is a contradiction as $\mu<1$.

For the mean ergodic properties of $\mathrm{C}$ acting in the Banach spaces $A^{-\gamma}$, for $\gamma>0$, we refer to Section 2 of [3].

Concerning the dynamics of $C$ recall that $T \in \mathcal{L}(X)$, with $X$ a separable lcHs, is hypercyclic if there exists $x \in X$ such that the orbit $\left\{T^{n} x: n \in \mathbb{N}_{0}\right\}$ is dense in $X$. If, for some $z \in X$, the projective orbit $\left\{\lambda T^{n} z: \lambda \in \mathbb{C}, n \in\right.$ $\left.\mathbb{N}_{0}\right\}$ is dense in $X$, then $T$ is called supercyclic. Clearly, hypercyclicity implies supercyclicity.

Proposition 3.5. The Cesàro operator $\mathrm{C}$ is not supercyclic in all of the spaces $A_{+}^{-\gamma}, \gamma \geq 0$, and $A_{-}^{-\gamma}, 0<\gamma \leq \infty$.

Proof. For the weight $\alpha:=(n)_{n \in \mathbb{N}}$, in which case the power series space $\Lambda_{0}(\alpha) \simeq H(\mathbb{D})$, it is proved in [5, Proposition 3.5] that $\mathrm{C}$ is not supercyclic on $H(\mathbb{D})$. Since all of the listed spaces are dense and continuously included in $H(\mathbb{D})$, the supercyclicity of any one of them would imply that $\mathrm{C} \in \mathcal{L}(H(\mathbb{D}))$ is supercyclic. 


\section{Non-Nuclearity of $A_{+}^{-\gamma}$ AND $A_{-}^{-\gamma}$.}

That the Fréchet space $H(\mathbb{D})$ is nuclear is a classical fact, [29, Example 28.9(4)], [32, Theorem 6.4.2]. Concerning its canonically imbedded subspaces $A_{+}^{-\gamma}$ and $A_{-}^{-\gamma}$ we have the following result.

\section{Proposition 4.1. $\quad$ (i) The Korenblum space $A^{-\infty}$ is nuclear.}

(ii) Each Fréchet space $A_{+}^{-\gamma}$ for $0 \leq \gamma<\infty$, and each (DFS)-space $A_{-}^{-\gamma}$, for $0<\gamma<\infty$, is a Schwartz space but, fails to be nuclear.

Concerning the proof of Proposition 4.1 it was already argued in Section 1 that all the spaces listed in Proposition 4.1 are Schwartz spaces. So, we only need to establish the claims related to nuclearity.

In relation to part (i) of Proposition 4.1, the Korenblum space $A^{-\infty}$ is known to be isomorphic to the strong dual of the Fréchet space $A^{\infty}(\overline{\mathbb{D}})$ consisting of all analytic functions on $\mathbb{D}$ which are $C^{\infty}$ on $\overline{\mathbb{D}}$; see, e.g., [12], [30]. Since $A^{\infty}(\overline{\mathbb{D}})$ is isomorphic to the nuclear Fréchet space $s$ of all rapidly decreasing sequences, it follows that $A^{-\infty}$ is nuclear.

The proof of the non-nuclearity of the spaces in part (ii) of Proposition 4.1 proceeds via a series of steps and relies on an important result of $\mathrm{T}$. Domenig, [19], [20]. In order to formulate this result we first require some preliminaries.

Consider the following dyadic lattice $\eta=\left(\eta_{k}\right)_{k \in \mathbb{N}_{0}}$ and its associated partition $\left(Q_{k}\right)_{k \in \mathbb{N}_{0}}$ of $\mathbb{D}$, [19, p.16], [20, p.329]. For each $n \in \mathbb{N}_{0}$ define the $2^{n}$ complex numbers

$$
\eta_{k}:=\left(1-2^{-n}\right) \exp \left(2 \pi i\left(k-2^{n}+\frac{1}{2}\right) 2^{-n}\right), \quad 2^{n} \leq k<2^{n+1},
$$

which then generate the associated $2^{n}$ polar rectangles

$Q_{k}:=\left\{w \in \mathbb{D}:\left|\eta_{k}\right| \leq|w| \leq \frac{1+\left|\eta_{k}\right|}{2},-\pi\left(1-\left|\eta_{k}\right|\right) \leq\left(\arg (w)-\arg \left(\eta_{k}\right)\right)<\pi\left(1-\left|\eta_{k}\right|\right)\right\}$,

for each $2^{n} \leq k<2^{n+1}$. Given any pair of numbers $s, t>0$, let $\xi(s, t)=$ $\left(\xi_{k}(s, t)\right)_{k \in \mathbb{N}_{0}}$ be the positive sequence given by

$$
\xi_{k}(s, t):=\left(1-\left|\eta_{k}\right|^{2}\right)^{-s} \sup _{w \in Q_{k}}\left(1-|w|^{2}\right)^{t}, \quad k \in \mathbb{N}_{0},
$$

and define the equivalent sequence $\xi^{*}(s, t)=\left(\xi_{k}^{*}(s, t)\right)_{k \in \mathbb{N}_{0}}$ by

$$
\xi_{k}^{*}(s, t):=\left(1-\left|\eta_{k}\right|\right)^{-s} \sup _{w \in Q_{k}}(1-|w|)^{t}, \quad k \in \mathbb{N}_{0} .
$$

Setting $\varphi(z):=z$, for $z \in \mathbb{D}$, the sequence $\xi(s, t)$ given by (4.2) is precisely the sequence $\xi$ defined in [19, p.53], [20, p.348]. The equivalence of $\xi(s, t)$ and $\xi^{*}(s, t)$ is immediate from the inequalities

$$
1-|z| \leq 1-|z|^{2}=(1+|z|)(1-|z|) \leq 2(1-|z|), \quad z \in \mathbb{D},
$$

which imply that

$$
2^{-s} \xi_{k}^{*}(s, t) \leq \xi_{k}(s, t) \leq 2^{t} \xi_{k}^{*}(s, t), \quad k \in \mathbb{N}_{0}, \quad s, t>0 .
$$


Lemma 4.2. Let $0<s<t$. Then, for each $n \in \mathbb{N}_{0}$, we have

$$
\frac{1}{2^{t}} \cdot \frac{1}{\left(2^{n}\right)^{t-s}} \leq \xi_{k}^{*}(s, t) \leq \frac{1}{\left(2^{n}\right)^{t-s}}, \quad 2^{n} \leq k<2^{n+1} .
$$

Proof. Fix $n \in \mathbb{N}_{0}$. It follows from (4.1) that $\left|\eta_{k}\right|=1-2^{-n}$ and hence, that $1-\frac{\left(1+\left|\eta_{k}\right|\right)}{2}=2^{-(n+1)}$ for all $2^{n} \leq k<2^{n+1}$. It is then clear from the definition of $Q_{k}$ that

$$
2^{-(n+1)} \leq 1-|w| \leq 2^{-n}, \quad w \in Q_{k}, \quad 2^{n} \leq k<2^{n+1},
$$

which in turn implies that

$$
2^{-(n+1) t} \leq \sup _{w \in Q_{k}}(1-|w|)^{t} \leq 2^{-n t}, \quad 2^{n} \leq k<2^{n+1} .
$$

Since $\left(1-\left|\eta_{k}\right|\right)=2^{-n}$ for $2^{n} \leq k<2^{n+1}$, it follows from the previous inequality and (4.3) that

$$
\left(2^{-n}\right)^{-s}\left(2^{-(n+1) t}\right) \leq \xi_{k}^{*}(s, t) \leq\left(2^{-n}\right)^{-s} 2^{-n t}, \quad 2^{n} \leq k<2^{n+1},
$$

which is precisely (4.6).

Remark 4.3. (i) The inequalities (4.5) and (4.6) show, for each pair $0<$ $s<t$, that both sequences $\xi(s, t)$ and $\xi^{*}(s, t)$ are bounded. Hence, each diagonal operator $D_{\xi(s, t)}$ (resp. $\left.D_{\xi^{*}(s, t)}\right)$ defined by mapping $x=\left(x_{k}\right)_{k \in \mathbb{N}_{0}} \in$ $\ell_{\infty}$ to $\left(\xi_{k}(s, t) x_{k}\right)_{k \in \mathbb{N}_{0}}$ (resp. to $\left.\left(\xi_{k}^{*}(s, t) x_{k}\right)_{k \in \mathbb{N}_{0}}\right)$ belongs to $\mathcal{L}\left(\ell_{\infty}\right)$ and maps the closed subspace $c_{0} \subseteq \ell_{\infty}$ into $c_{0}$.

(ii) Given a sequence $\xi \in \mathbb{C}^{\mathbb{N}_{0}}$, the diagonal operator $D_{\xi}: \mathbb{C}^{\mathbb{N}_{0}} \rightarrow \mathbb{C}^{\mathbb{N}_{0}}$ maps $\ell_{\infty}$ into $\ell_{\infty}$ if and only if $\xi \in \ell_{\infty}$. We will require the fact that $D_{\xi} \in \mathcal{L}\left(\ell_{\infty}\right)$ is nuclear if and only if $\xi \in \ell_{1}$. Indeed, if $\xi \in \ell_{1}$, then $D_{\xi}: \ell_{1} \rightarrow \ell_{1}$ is nuclear, [32, p.54]. Since $D_{\xi}: \ell_{\infty} \rightarrow \ell_{\infty}$ is the dual operator of $D_{\xi}: \ell_{1} \rightarrow \ell_{1}$, it is also nuclear, [32, Proposition 3.1.8]. Conversely, suppose that $D_{\xi} \in \mathcal{L}\left(\ell_{\infty}\right)$ is nuclear. Let $J: c_{0} \rightarrow \ell_{\infty}$ be the (continuous) canonical inclusion map. Then the restriction $\left.D_{\xi}\right|_{c_{0}}: c_{0} \rightarrow \ell_{\infty}$, being equal to $D_{\xi} \circ J$, is also nuclear by the ideal property of nuclear operators. Since $\left.D_{\xi}\right|_{c_{0}}$ takes its values in $c_{0}$, it follows that $D_{\xi}: c_{0} \rightarrow c_{0}$ is nuclear and hence, so is its dual operator $D_{\xi}: \ell_{1} \rightarrow \ell_{1}$. In particular, $\xi \in \ell_{1}$, [32, p.54].

To formulate the relevant result of Domenig we require some further notation. First, for $s>0$, we note that the Banach space

$$
X_{s}:=\left\{f \in H(\mathbb{D}):\|f\|_{X_{s}}:=\sup _{z \in \mathbb{D}}\left(1-|z|^{2}\right)^{s}|f(s)|<\infty\right\}
$$

as defined in [19, p.20], [20, p.348], coincides with $A^{-s}$ as defined in Section 1 above and, due to (4.4), the norms $\|\cdot\|_{X_{s}}$ and $\|\cdot\|_{-s}$ are equivalent. Moreover, for the particular analytic function $\varphi(z):=z$, for $z \in \mathbb{D}$, continuity of the composition operator $C_{\varphi}: X \rightarrow Y$, acting between Banach spaces of analytic functions $X, Y$ which are continuously included in $H(\mathbb{D})$, is equivalent to $X$ being continuously contained in $Y$. Finally, recalling that the ideal of absolutely summing operators is maximal, [18, Remark 6.18], we obtain (for $p=q=\infty$ ) the following special case of Theorem 4.2 in [19]. 
Proposition 4.4. Let $0<s<t$, in which case $A^{-s} \subseteq A^{-t}$ continuously. The following statements are equivalent.

(i) The canonical inclusion $A^{-s} \subseteq A^{-t}$ is an absolutely summing operator.

(ii) The diagonal operator $D_{\xi(s, t)}: \ell_{\infty} \rightarrow \ell_{\infty}$ is absolutely summing.

We can now present the proof of Proposition 4.1(ii). We begin with the Fréchet spaces $A_{+}^{-\gamma}$.

So, fix $\gamma \in[0, \infty)$. Suppose that $A_{+}^{-\gamma}=\operatorname{proj}_{n} A^{-\left(\gamma+\frac{1}{n}\right)}$ is nuclear. Set $r:=\left(\gamma+\frac{1}{2}\right)>0$. By the criterion for nuclearity of a projective limit there exists $m \in \mathbb{N}$ with $m>2$ such that $t:=\left(\gamma+\frac{1}{m}\right) \in(\gamma, r)$ and the inclusion map $A^{-t} \subseteq A^{-r}$ is nuclear, [29, Proposition 28.4], [32, Section 4.1.2]. In particular, this inclusion map is then also absolutely summing. Since $0<t<r$, the diagonal operator $D_{\xi(t, r)}: \ell_{\infty} \rightarrow \ell_{\infty}$ is absolutely summing (cf. Proposition 4.4). By the same reasoning there exists $n \in \mathbb{N}$ with $n>m$ such that $s:=\left(\gamma+\frac{1}{n}\right) \in(\gamma, t)$ and the inclusion map $A^{-s} \subseteq A^{-t}$ is absolutely summing. Since $0<s<t$, Proposition 4.4 again implies that $D_{\xi(s, t)}: \ell_{\infty} \rightarrow \ell_{\infty}$ is also absolutely summing. By Theorem 3.3.5 of [32, p.66], the diagonal operator $D_{\xi(s, t) \xi(t, r)}=D_{\xi(s, t)} D_{\xi(t, r)} \in \mathcal{L}\left(\ell_{\infty}\right)$ is nuclear. Then Remark 4.3(ii) implies that $\sum_{k=0}^{\infty} \xi_{k}(s, t) \xi_{k}(t, r)<\infty$. According to (4.5) also $\sum_{k=0}^{\infty} \xi_{k}^{*}(s, t) \xi_{k}^{*}(t, r)<\infty$. But, for $n \in \mathbb{N}_{0}$ fixed, it follows from Lemma 4.2 that

$$
\sum_{k=2^{n}}^{2^{n+1}-1} \xi_{k}^{*}(s, t) \xi_{k}^{*}(t, r) \geq 2^{n} \cdot \frac{1}{2^{t}} \cdot \frac{1}{\left(2^{n}\right)^{t-s}} \cdot \frac{1}{2^{r}} \cdot \frac{1}{\left(2^{n}\right)^{r-t}}=\frac{1}{2^{r+t}} \cdot\left(2^{n}\right)^{1-(r-s)} .
$$

Since $0 \leq \gamma<s<t<r=\left(\gamma+\frac{1}{2}\right)$ implies that $0<(r-s)<\frac{1}{2}<1$, we can conclude that the series

$$
\sum_{k=0}^{\infty} \xi_{k}^{*}(s, t) \xi_{k}^{*}(t, r)=\sum_{n=0}^{\infty}\left(\sum_{k=2^{n}}^{2^{n+1}-1} \xi_{k}^{*}(s, t) \xi_{k}^{*}(t, r)\right) \geq \frac{1}{2^{r+t}} \sum_{n=0}^{\infty}\left(2^{n}\right)^{1-(r-s)}
$$

diverges; contradiction. Hence, $A_{+}^{-\gamma}$ cannot be nuclear.

For the inductive limit spaces $A_{-}^{-\gamma}$ we use the criterion that a complete (LB)-space $F=\operatorname{ind}_{n} F_{n}$ is nuclear if and only if it is dual nuclear if and only if for every $n \in \mathbb{N}$ there exists $m>n$ such that the imbedding $F_{n} \hookrightarrow F_{m}$ is nuclear; see, for example, [25, Section 21.5], [29, p.356], [32, Proposition 4.1.6 and Theorem 4.3.3]. So, fix $\gamma \in(0, \infty)$ and suppose that $A_{-}^{-\gamma}=$ ind ${ }_{n \geq n_{0}} A^{-\left(\gamma-\frac{1}{n}\right)}$ is nuclear with $n_{0} \in \mathbb{N}$ chosen so that $\left(\gamma-\frac{1}{n}\right)>0$ for all $n \geq n_{0}$. Select $s \in(\beta, \gamma)$ where $\beta:=\max \left\{0,\left(\gamma-\frac{1}{2}\right)\right\}$. Then there exists $m \in \mathbb{N}$ such that $t:=\left(\gamma-\frac{1}{m}\right) \in(s, \gamma)$ with the inclusion map $A^{-s} \subseteq A^{-t}$ being nuclear hence, also absolutely summing. According to Proposition 4.4 the diagonal operator $D_{\xi(s, t)} \in \mathcal{L}\left(\ell_{\infty}\right)$ is absolutely summing. By the same reasoning again there exists $n \in \mathbb{N}$ such that $r:=\left(\gamma-\frac{1}{n}\right) \in(t, \gamma)$ and the inclusion map $A^{-t} \subseteq A^{-r}$ is absolutely summing, which implies 
(via Proposition 4.4) that also $D_{\xi(t, r)}$ is absolutely summing. Arguing as in the previous paragraph it follows that $\sum_{k=0}^{\infty} \xi_{k}^{*}(s, t) \xi_{k}^{*}(t, r)<\infty$. But, the inequalities $0 \leq \beta<s<t<r<\gamma$ imply that $0<(r-s)<\frac{1}{2}<1$ and so the estimate (4.7) again provides a contradiction. Accordingly, $A_{-}^{-\gamma}$ cannot be nuclear.

The proof of Proposition 4.1 is thereby complete.

Remark 4.5. The two step argument used in the proof of Proposition 4.1(ii), i.e., to produce a pair of absolutely summing operators $D_{\xi(s, t)}$ and $D_{\xi(t, r)}$ whose product is then nuclear, is necessary because Proposition 4.4 is not directly applicable to the ideal of nuclear operators itself as this ideal, in general, is not maximal, [18, Corollary 6.21].

\section{Appendix. Abstract spectral Results.}

The following result concerning the spectrum of operators on Fréchet spaces is Lemma 2.1 of [4].

Lemma 5.1. Let $X=\cap_{n \in \mathbb{N}} X_{n}$ be a Fréchet space which is the intersection of a sequence of Banach spaces $\left(\left(X_{n},\|\cdot\|_{n}\right)\right)_{n \in \mathbb{N}}$ satisfying $X_{n+1} \subseteq X_{n}$ with $\|x\|_{n} \leq\|x\|_{n+1}$ for each $n \in \mathbb{N}$ and $x \in X_{n+1}$. Let $T \in \mathcal{L}(X)$ satisfy the following condition:

(A) For each $n \in \mathbb{N}$ there exists $T_{n} \in \mathcal{L}\left(X_{n}\right)$ such that the restriction of $T_{n}$ to $X$ (resp. of $T_{n}$ to $\left.X_{n+1}\right)$ coincides with $T$ (resp. with $\left.T_{n+1}\right)$.

Then $\sigma(T ; X) \subseteq \cup_{n \in \mathbb{N}} \sigma\left(T_{n} ; X_{n}\right)$ and $R(\lambda, T)$ coincides with the restriction of $R\left(\lambda, T_{n}\right)$ to $X$ for each $n \in \mathbb{N}$ and $\lambda \in \cap_{n \in \mathbb{N}} \rho\left(T_{n} ; X_{n}\right)$.

Moreover, if $\cup_{n \in \mathbb{N}} \sigma\left(T_{n} ; X_{n}\right) \subseteq \overline{\sigma(T ; X)}$, then

$$
\sigma^{*}(T ; X)=\overline{\sigma(T ; X)} \text {. }
$$

An analogue of Lemma 5.1 for (LB)-spaces is as follows.

Lemma 5.2. Let $E=\operatorname{ind}_{n}\left(E_{n},\|\cdot\|_{n}\right)$ be a Hausdorff inductive limit of Banach spaces. Let $T \in \mathcal{L}(E)$ satisfy the following condition:

(A) For each $n \in \mathbb{N}$ the restriction $T_{n}$ of $T$ to $E_{n}$ maps $E_{n}$ into itself and $T_{n} \in \mathcal{L}\left(E_{n}\right)$.

Then the following properties are satisfied.

(i) $\sigma_{p t}(T ; E)=\cup_{n \in \mathbb{N}} \sigma_{p t}\left(T_{n} ; E_{n}\right)$.

(ii) $\sigma(T ; E) \subseteq \cap_{m \in \mathbb{N}}\left(\cup_{n=m}^{\infty} \sigma\left(T_{n} ; E_{n}\right)\right)$. Moreover, if $\lambda \in \cap_{n=m}^{\infty} \rho\left(T_{n} ; E_{n}\right)$ for some $m \in \mathbb{N}$, then $R\left(\lambda, T_{n}\right)$ coincides with the restriction of $R(\lambda, T)$ to $E_{n}$ for each $n \geq m$.

(iii) If $\cup_{n=m}^{\infty} \sigma\left(T_{n} ; E_{n}\right) \subseteq \overline{\sigma(T ; E)}$ for some $m \in \mathbb{N}$, then

$$
\sigma^{*}(T ; E)=\overline{\sigma(T ; E)} .
$$

Proof. (i) Let $\lambda \in \sigma_{p t}(T ; E)$. Then there exists $x \in E \backslash\{0\}$ such that $T x=$ $\lambda x$. Since $E=\operatorname{ind}_{n}\left(E_{n},\|\cdot\|_{n}\right)$, in particular $E=\cup_{n=1}^{\infty} E_{n}$, and condition 
(A) holds, there exists $n \in \mathbb{N}$ such that $x \in E_{n} \backslash\{0\}$ and $T_{n} x=T x=\lambda x$. Accordingly, $\lambda \in \sigma_{p t}\left(T_{n} ; E_{n}\right)$.

Conversely, fix $n \in \mathbb{N}$ and let $\lambda \in \sigma_{p t}\left(T_{n} ; E_{n}\right)$. Then there exists $x \in$ $E_{n} \backslash\{0\}$ such that $T_{n} x=\lambda x$. Since $E=\operatorname{ind}_{n}\left(E_{n},\|\cdot\|_{n}\right)$ and condition (A) holds, $x \in E \backslash\{0\}$ and $T x=T_{n} x=\lambda x$, that is, $\lambda \in \sigma_{p t}(T ; E)$.

(ii) Fix $\lambda \notin \cap_{m \in \mathbb{N}}\left(\cup_{n=m}^{\infty} \sigma\left(T_{n} ; E_{n}\right)\right)$. Then there exists $m \in \mathbb{N}$ such that $\lambda \notin \cup_{n=m}^{\infty} \sigma\left(T_{n} ; E_{n}\right)$, that is, $\lambda \in \cap_{n=m}^{\infty} \rho\left(T_{n} ; E_{n}\right)$. This implies that the operator $(\lambda I-T): E \rightarrow E$ is bijective. To see this, we proceed as follows.

To show that $(\lambda I-T): E \rightarrow E$ is injective, suppose that $(\lambda I-T) x=0$ for some $x \in E$. Then $x \in E_{n_{0}}$ for some $n_{0} \in \mathbb{N}$ with $n_{0} \geq m$ (recall that $E_{n} \subseteq E_{n+1} \subseteq E$ for all $\left.n \in \mathbb{N}\right)$ and so, condition (A) yields $\left(\lambda I-T_{n_{0}}\right) x=0$ in $E_{n_{0}}$. Since $\lambda \in \rho\left(T_{n_{0}} ; E_{n_{0}}\right)$, this implies that $x=0$.

To check that $(\lambda I-T): E \rightarrow E$ is surjective, fix $y \in E$. Then $y \in E_{n_{0}}$ for some $n_{0} \in \mathbb{N}$ with $n_{0} \geq m$. Since $\lambda \in \rho\left(T_{n_{0}} ; E_{n_{0}}\right)$, there exists $x \in E_{n_{0}} \subseteq E$ satisfying $\left(\lambda I-T_{n_{0}}\right) x=y$ in $E_{n_{0}}$. By condition (A) we have $y=(\lambda I-T) x$ in $E$.

Finally, since $E$ is an inductive limit of Banach spaces and $(\lambda I-T) \in$ $\mathcal{L}(E)$, the open mapping theorem is valid and so we can conclude that $\lambda \in \rho(T ; E)$.

Condition (A) implies, for each $n \in \mathbb{N}$ and $\lambda \in \mathbb{C}$, that the restriction $\lambda I-T_{n}$ of $\lambda I-T$ to $E_{n}$ maps $E_{n}$ into itself and $\lambda I-T_{n} \in \mathcal{L}\left(E_{n}\right)$. The second assertion in (ii) follows from this observation.

(iii) Since $\sigma(T ; E) \subseteq \sigma^{*}(T ; E)$ with $\sigma^{*}(T ; E)$ closed in $\mathbb{C}$, we always have $\overline{\sigma(T ; E)} \subseteq \sigma^{*}(T ; E)$. So, it remains to show the reverse inclusion.

If $\overline{\sigma(T ; E)}=\mathbb{C}$, then there is nothing to verify. So, suppose there exists $\lambda \in \mathbb{C} \backslash \overline{\sigma(T ; E)}$. Then there exists $\varepsilon>0$ such that $B(\lambda, \varepsilon) \cap \overline{\sigma(T ; E)}=\emptyset$. By the assumption of (iii) we have $B(\lambda, \varepsilon) \subseteq \rho\left(T_{n} ; E_{n}\right)$ for each $n \geq m$. Suppose there exists $x \in E$ such that $A:=\{R(\mu, T) x: \mu \in B(\lambda, \varepsilon)\} \notin \mathcal{B}(E)$. Since $x \in E_{n_{0}}$ for some $n_{0} \in \mathbb{N}$ with $n_{0} \geq m$ and $R\left(\lambda, T_{n_{0}}\right)$ coincides with the restriction of $R(\lambda, T)$ to $E_{n_{0}}$ (by (ii)), it follows that $A\left(n_{0}\right):=$ $\left\{R\left(\mu, T_{n_{0}}\right) x: \mu \in B(\lambda, \varepsilon)\right\} \notin \mathcal{B}\left(E_{n_{0}}\right)$ (as $E_{n_{0}}$ is continuously included in $E$ ). But, $B(\lambda, \varepsilon) \subseteq \rho\left(T_{n_{0}} ; E_{n_{0}}\right)$ with $E_{n_{0}}$ a Banach space implies that $A\left(n_{0}\right) \in$ $\mathcal{B}\left(E_{n_{0}}\right)$; contradiction. Hence, $\{R(\mu, T): \mu \in B(\lambda, \varepsilon)\}$ is a bounded subset of $\mathcal{L}_{s}(E)$, i.e., an equicontinuous subset of $\mathcal{L}(E)$, [29, Propositions $23.27 \&$ 24.16]. Accordingly, $\lambda \in \rho^{*}(T ; E)$.

Acknowledgements. The research of the first two authors was partially supported by the projects MTM2013-43540-P and MTM2016-76647-P. The second author gratefully acknowledges the support of the Alexander von Humboldt Foundation.

\section{REFERENCES}

[1] A.A. Albanese, J. Bonet, W.J. Ricker, Mean ergodic operators in Fréchet spaces, Ann. Acad. Sci. Fenn. Math. 34 (2009), 401-436. 
[2] A.A. Albanese, J. Bonet, W.J. Ricker, Montel resolvents and uniformly mean ergodic semigroups of linear operators, Quaest. Math. 36 (2013), 253-290.

[3] A.A. Albanese, J. Bonet, W.J. Ricker, The Cesàro operator in growth Banach spaces of analytic functions, Integral Equat. Oper. Theory 86 (2016), 97-112.

[4] A.A. Albanese, J. Bonet, W.J. Ricker, The Cesàro operator in the Fréchet spaces $\ell^{p+}$ and $L^{p-}$, Glasgow Math. J., DOI: 10.1017/S00178951600015X.

[5] A.A. Albanese, J. Bonet, W.J. Ricker, The Cesàro operator on power series spaces, Studia Math. (to appear).

[6] A. Aleman, A class of integral operators on spaces of analytic functions, in: Proceedings of the Winter School in Operator Theory and Complex Analysis, Univ. Málaga Secr. Publ., Málaga, 2007, pp. 3-30.

[7] A. Aleman, O. Constantin, Spectra of integration operators on weighted Bergman spaces, J. Anal. Math. 109 (2009), 199-231.

[8] A. Aleman, J.A. Peláez, Spectra of integration operators and weighted square functions, Indiana Univ. Math. J. 61 (2012), 1-19.

[9] A. Aleman, A.-M. Persson, Resolvent estimates and decomposable extensions of generalized Cesàro operators, J. Funct. Anal. 258 (2010), 67-98.

[10] A. Aleman, A.G. Siskakis, An integral operator on $H^{p}$, Complex Var. Theory Appl. 28 (1995), 149-158.

[11] A. Aleman, A.G. Siskakis, Integration operators on Bergman spaces, Indiana Univ. Math. J. 46 (1997), 337-356.

[12] D.E. Barrett, Duality between $A^{\infty}$ and $A^{-\infty}$ on domains with nondegenerate corners, Multivariable operator theory (Seattle, WA, 1993), pp.77-87, Contemporary Math. 185, Amer. Math. Soc., Providence, 1995.

[13] K.D. Bierstedt, J. Bonet, A. Galbis, Weighted spaces of holomorphic functions on bounded domains, Michigan Math. J. 40 (1993), 271-297.

[14] K.D. Bierstedt, J. Bonet, J. Taskinen, Associated weights and spaces of holomorphic functions, Studia Math. 127 (1998), 137-168.

[15] K.D. Bierstedt, R. Meise, W. H. Summers, A projective description of weighted inductive limits, Trans. Amer. Math. Soc. 272 (1982), 107-160.

[16] K.D. Bierstedt, W. H. Summers, Biduals of weighted Banach spaces of analytic functions, J. Austral. Math. Soc. (Series A) 54 (1993), 70-79.

[17] J. Bonet, P. Domański, M. Lindström, J. Taskinen, Composition operators between weighted Banach spaces of analytic functions, J. Austral. Math. Soc. (Series A),64 (1998), 101-118.

[18] J. Diestel, H. Jarchow, A. Tonge, Absolutely Summing Operators, Cambridge University Press, Cambridge, 1995.

[19] T. Domenig, Composition Operators on Weighted Bergman Spaces and Hardy Spaces. Atomic Decompositions and Diagonal Operators, Ph.D. Thesis, University of Zürich, 1997. [Zbl 0909.47025].

[20] T. Domenig, Composition operators belonging to operator ideals, J. Math. Anal. Appl., 237 (1999), 327-349.

[21] N. Dunford, J.T. Schwartz, Linear Operators I: General Theory. 2nd Printing, Wiley Interscience Publ., New York, 1964.

[22] R.E. Edwards, Functional Analysis. Theory and Applications, Holt, Rinehart and Winston, New York Chicago San Francisco, 1965.

[23] A. Grothendieck, Topological Vector Spaces, Gordon and Breach, London, 1973.

[24] H. Hedenmalm, B. Korenblum, K. Zhu, Theory of Bergman Spaces. Grad. Texts in Math. 199, Springer-Verlag, New York, 2000.

[25] H. Jarchow, Locally Convex Spaces, Teubner, Stuttgart, 1981. 
[26] B. Korenblum, An extension of the Nevanlinna theory, Acta Math. 135 (1975), $187-219$.

[27] U. Krengel, Ergodic Theorems. de Gruyter Studies in Mathematics, 6. Walter de Gruyter Co., Berlin, 1985.

[28] W. Lusky, On the isomorphism classes of weighted spaces of harmonic and holomorphic functions, Studia Math. 175 (1) (2006), 19-40.

[29] R. Meise, D. Vogt, Introduction to Functional Analysis. Clarendon Press, Oxford, 1997.

[30] S.N. Melikhov, (DFS )-spaces of holomorphic functions invariant under differentiation, J. Math. Anal. Appl. 297 (2004), 577-586.

[31] A.-M. Persson, On the spectrum of the Cesàro operator on spaces of analytic functions. J. Math. Anal Appl. 340 (2008), 1180-1203.

[32] A. Pietsch, Nuclear Locally Convex Spaces, Springer, Berlin Heidelberg, 1972.

[33] A.L. Shields, D.L. Williams, Bounded projections, duality and multipliers in spaces of analytic functions, Trans. Amer. Math. Soc. 162 (1971), 287-302.

[34] A. Siskakis, Volterra operators on spaces of analytic functions - a survey, in: Proceedings of the First Advanced Course in Operator Theory and Complex Analysis, Univ. Sevilla Serc. Publ., Seville, 2006, pp. 51-68.

Angela A. Albanese, Dipartimento di Matematica e Fisica "E. De Giorgi", Università del Salento- C.P.193, I-73100 Lecce, Italy

E-mail address: angela.albanese@unisalento.it

José Bonet, Instituto Universitario de Matemática Pura y Aplicada

iUMPA, Universitat Politècnica de València, E-46071 Valencia, Spain

E-mail address: jbonet@mat.upv.es

Werner J. Ricker, Math.-Geogr. Fakultät, Katholische Universität EichstättIngOlSTAdT, D-85072 EichStätt, Germany

E-mail address: werner.ricker@ku-eichstaett.de 